\title{
Deconstructing the Spectrum of the Soft X-ray Background
}

\author{
K. D. KUNTZ1 \\ Department of Astronomy, University of Maryland, College Park, MD 20742 \\ S. L. SNOWDEN ${ }^{2,3}$ \\ Code 662, NASA/Goddard Space Flight Center, Greenbelt, MD 20771.
}

\begin{abstract}
The soft X-ray background in the $0.1-1.0 \mathrm{keV}$ band is known to be produced by at least three sources; the Local Hot Bubble (LHB), the extragalactic power law (EPL), and a seemingly galactic component that lies outside the bulk of the absorption that is due to the ISM of the galactic disk. This last component, which we call the Trans-Absorption Emission (TAE), has been modeled by a number of groups who have derived disparate measures of its temperature. The differences have arisen from differing assumptions about the structure of the emitting gas and unrecognized methodological difficulties. In particular, spectral fitting methods do not uniquely separate the TAE from the foreground emission that is due the LHB. This "degeneracy" can be resolved using the angular variation of the absorption of the TAE.

We show that the TAE cannot be characterized by a single thermal component; no singlecomponent model can be consistent with both the spectral energy distribution of the TAE emission and the angular variation due to absorption by the galactic disk. We use the angular anticorrelation of the ROSAT All-Sky Survey with the galactic absorption to separate local from distant emission components, and to fit the spectral energy distribution of the resulting distant emission. We find that the emission is best described by a two-thermal-component model with $\log T_{S}=6.06_{-0.17}^{+0.13}$ and $\log T_{H}=6.42_{-0.12}^{+0.14}$. This two-thermal-component TAE fits the ROSAT spectral energy distribution significantly better than single-component models, and is consistent with both angular variation and spectral constraints.
\end{abstract}

Subject headings: galaxies: Milky Way - interstellar: matter - X-rays: galaxies,general

\section{Introduction}

The canonical model of the soft X-ray background (0.1-1.0 keV) posits a Local Cavity (in the $\mathrm{H}$ I of the galactic disk) that is filled, at least in part, by hot gas emitting mostly in the $\frac{1}{4} \mathrm{keV}$ band. This region of $\mathrm{X}$-ray emitting gas is known as the Local Hot Bubble (LHB). The LHB emission is absorbed by the nearby very tenuous Local Interstellar Cloud (LIC), otherwise known as the Local Fluff. Beyond the Local Cavity there are

\footnotetext{
${ }^{1}$ E-mail-1:kuntz@astro.umd.edu

${ }^{2}$ E-mail-I:snowden@lheavx.gsfc.nasa.gov

${ }^{3}$ Universities Space Research Association
}

thicker layers of absorbing gas which partially absorb emission from an isotropic extragalactic component and emission from at least one other component. This latter component we will call the "trans-absorption emission" (TAE) or the "distant component". The local component and the distant component are usually modeled as thermal plasmas. The isotropic extragalactic component, which is most clearly seen at $\mathrm{E}>1 \mathrm{keV}$, and is presumably due to the accumulation of unresolved point sources, is modeled as a power-law, and will be referred to as the Extragalactic Power Law (EPL). The distant component is required to contribute to the observed excess over the extrapolation of the EPL in both the $\frac{1}{4} \mathrm{keV}$ and $\frac{3}{4} \mathrm{keV}$ 
bands.

Table 1 contains a compendium of measures of the temperature of the thermal components using various techniques. The ROSAT measures alone show a wide dispersion. In particular, PSPC spectral fitting produces lower LHB temperatures and higher halo temperatures than do early broadband measures and PSPC broad-band deabsorption measures. In order to understand the source of this difference, the next section assumes the canonical model and explores the limitations of the methods used for determining the parameters of that model.

We find that although a portion of the difference is due to inadequacies in the spectral fitting method, the bulk of the difference is due to assumptions about the nature of the distant component. Most investigators have assumed that the TAE is due to a single-temperature galactic halo. We find that none of the existing single-component models satisfies all of the constraints placed upon the data by the angular variation of the absorption of the TAE.

We attempt to determine the thermal structure of the TAE by combining the all-sky deabsorption method of Snowden et al. (1998) with six-band Spectral Energy Distribution (SED) fitting. Section 3 describes the assumptions, input data, and limitations of our method. We find that although the data require more than one thermal component to describe the TAE, the data are sufficient to characterize only two components, though a greater number may exist, and the temperature of these components may vary with position. Section 4 describes the results and finds the temperatures of the two thermal components. Section 5 summarizes our arguments and discusses the implications of a two-component TAE.

\section{Analysis of Previous Results}

Using the canonical model, several efforts have been made to determine the spectrum of the soft $\mathrm{X}$-ray background. We divide these methods into two groups: spectral fitting of individual PSPC pointings, and all-sky deabsorption of broad-band images. In both of these cases, the data were fit to the function

$$
I_{O}=I_{L} e^{-\left(N_{f}\right) \sigma\left(\nu, N_{f}, \mathcal{S}\right)}
$$

$$
\begin{aligned}
& +I_{D} e^{-\left(N_{b}\right) \mathcal{\sigma}\left(\nu, N_{b}, \mathcal{S}\right)} \\
& +I_{E} e^{-\left(N_{b}\right) \sigma\left(\nu, N_{b}, \mathcal{S}\right)}
\end{aligned}
$$

where $I_{O}$ is the observed X-ray flux. $I_{L}$ is the local (LHB) X-ray emission which may be absorbed by an intervening layer of column $\Lambda_{f}, I_{D}$ is the distant emission (TAE) and $I_{E}$ is the contribution due to the EPL. Both the distant and extragalactic components are absorbed by a column $N_{b}$. The quantity $\sigma$ is the absorption cross-section. In the case of spectral fitting, $\sigma$ is dependent only upon energy $(\nu)$, but for broad-band deabsorption we use the effective cross-section for a given band which is dependent both upon the magnitude of the absorbing column $(N)$, and upon the spectral shape of the background emission $(\mathcal{S})$.

\subsection{Spectral Fitting}

Several groups (e.g., Chen et al. 1997 and Miyaji et al. 1998) have attempted to fit the spectrum of the X-ray background as observed in individual PSPC pointings. Attention has focused upon determining the index of the EPL; determination of the temperatures and normalizations of the two thermal components has been incidental. Due to this focus, the ROSAT PSPC data have usually been supplemented with $A S C A$ observations of the same field in order to gain a greater lever arm for fitting the EPL. Fitting the thermal components is more problematic.

The thermal components occupy only a very limited spectral region in the ROSAT range; the emission lies mostly within $0.1-0.4 \mathrm{keV}$, which is completely outside of the $A S C A$ range. At these energies, absorption, and the uncertainty due to its correction, is much greater. Also greater are the non-cosmic foregrounds; the Long Term Enhancement (LTE) and the Scattered Solar X-rays (SSX). The LTE is a particular problem for PSPC pointed observations as adequate removal requires a light curve extending over (at minimum) several weeks to months (Snowden et al. 1995, 1994b). Much of the disagreement between different spectral fits has been attributed to inadequate noncosmic background removal (Miyaji et al. 1998).

Inadequate spectral resolution is also a problem. There are only about five independent spectral resolution elements in the ROSAT PSPC energy band, and only three spanning the energies emitted by the thermal components. Thus, even 
TABLE 1

COMPENDIUM

\begin{tabular}{|c|c|c|c|c|c|c|}
\hline \multirow[t]{2}{*}{ Source } & \multicolumn{2}{|c|}{$T_{L}$} & \multicolumn{2}{|c|}{$T_{D 1}$} & \multicolumn{2}{|c|}{$T_{D 2}$} \\
\hline & $\mathrm{keV}$ & $\log T$ & $\mathrm{keV}$ & $\log T$ & $\mathrm{keV}$ & $\log T$ \\
\hline \multicolumn{7}{|c|}{ Pre-ROSAT Results } \\
\hline Block et al. (1986) & $0.077-0.097$ & $5.95-6.05$ & $\cdots$ & . & $\cdots$ & $\cdots$ \\
\hline Juda et al.(1991) & $0.054-0.136$ & $5.8-6.2$ & $\cdots$ & $\cdots$ & $\cdots$ & $\cdots$ \\
\hline Snowden et al. (1990) & 0.086 & 6.0 & $\cdots$ & $\cdots$ & $\cdots$ & $\cdots$ \\
\hline \multicolumn{7}{|c|}{ ROSAT Era Results } \\
\hline Chen, Fabian \& Gendreau(1997) & 0.05 & 5.76 & 0.12 & 6.14 & $\cdots$ & $\cdots$ \\
\hline $\operatorname{Kerp}(1994)$ & $0.061_{-0.015}^{+0.016}$ & $5.85 \pm 0.1$ & $0.172_{-0.035}^{+0.045}$ & $6.3 \pm 0.1$ & $\cdots$ & $\cdots$ \\
\hline Miyaji et al.(1998) & $.057-.09$ & $5.82-6.02$ & $0.142-0.148$ & $6.217-6.233$ & $\cdots$ & $\cdots$ \\
\hline Peitz et al. $(1998)^{b}$ & $\cdots$ & $\cdots$ & 0.135 & $6.195 \pm 0.05^{b}$ & $\cdots$ & $\cdots$ \\
\hline Sidher $(1996)^{c}$ & $\cdots$ & $\cdots$ & $0.137(0.216)$ & $6.2(6.4)^{\mathrm{c}}$ & $\cdots$ & $\cdots$ \\
\hline Snowden et al. $(1998)^{d}$ & $0.119_{-0.012}^{+0.034}$ & $6.14_{-0.05}^{+0.11} \mathrm{e} g$ & $0.106_{-0.018}^{+0.022}$ & $6.09_{-0.08}^{+0.08 \mathrm{e}}$ & $\cdots$ & $\cdots$ \\
\hline Snowden et al. $(2000)^{\mathrm{h}}$ & $0.119_{-0.024}^{+0.034}$ & $6.14_{-0.10}^{+0.110} \mathrm{e} g$ & $0.100_{-0.030}^{+0.033}$ & $6.07_{-0.16}^{+0.12 e}$ & $\cdots$ & $\cdots$ \\
\hline \multicolumn{7}{|c|}{ ROSAT Results - This Work } \\
\hline Hyades & $0.103_{-0.002}^{+0.002}$ & $6.08_{-0.01}^{+0.01 \mathrm{i}}$ & $\cdots$ & $\cdots$ & $\cdots$ & $\cdots$ \\
\hline Galactic Plane & $0.108_{-0.007}^{+0.005}$ & $6.11_{-0.03}^{+0.02} \mathrm{ei}$ & $\cdots$ & $\ldots$ & $\ldots$ & $\ldots$ \\
\hline Section 3.4 & $0.109_{-0.018}^{+0.047}$ & $6.10_{-0.08}^{+0.16} \mathrm{ef}^{\prime}$ & $0.098_{-0.031}^{0.035}$ & $6.06_{-0.17}^{+0.13 \mathrm{e}}$ & $0.227_{-0.055}^{+0.086}$ & $6.42_{-0.12}^{+0.14 \mathrm{e}}$ \\
\hline Section 4.1 .4 & $0.108 \pm 0.04$ & $6.10 \pm 0.02$ & $0.087 \pm 0.003$ & $6.00 \pm 0.02$ & $0.180 \pm 0.008$ & $6.32 \pm 0.02$ \\
\hline Section 4.1 .4 & $0.127 \pm 0.010$ & $6.17 \pm 0.03$ & $0.313 \pm 0.055$ & $6.56 \pm 0.07$ & $\cdots$ & $\ldots$ \\
\hline \multicolumn{7}{|c|}{ Post-ROSAT Results } \\
\hline Parmar et al. $(1999)^{\mathrm{k}}$ & $0.137_{-0.022}^{+0.011}$ & $6.2_{-0.07}^{+0.03}$ & $0.7_{-0.3}^{+0.4}$ & $6.91_{-0.24}^{+0.20 k}$ & $\cdots$ & 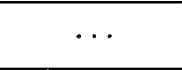 \\
\hline
\end{tabular}

${ }^{a}$ BB - Broad-Band Ratios, SF - Spectral Fitting, DA - De-Absorption

${ }^{\mathrm{b}}$ Temperature derived from the Mewe-Kaastra (Mewe et al. 1985) code. A fit of a Raymond \& Smith (1977) spectra to Kaastra spectrum absorbed by $1.5 \times 10^{20} \mathrm{~cm}^{-2}$ and folded through the ROSAT PSPC response yielded a Raymond \& Sm temperature of $\log T=6.18$. If a Mewe-Kaastra-Leidahl spectrum were used rather than a Mewe-Kaastra spectrum, the $R$ Smith plasma temperature would be $\log T=6.17$.

'Temperatures derived from the Landini \& Monsignori-Fossi (Landini \& Monsignori-Fossi 1990) code. Sidher et al. note tha' \& Smith code would produce a $\log T_{D}=6.4$.

dA second, hotter component is implied, but not explicitly characterized in this paper. The values given here are not quitt given in the original paper. Those values had been calculated from band ratios using an older detector response matrix; $u$. the current detector response matrix.

${ }^{e}$ Cited range is the FWHM of the distribution. These distributions are dominated by fit and/or data uncertainties; the c of temperatures is very narrow. See text $\$ 4$ for a fuller explanation.

${ }^{\mathrm{f}}$ The upper limit is not given by the upper FWHM of the distribution but by the highest R2/R1 ratio permitted by an $\mathrm{I}$ Raymond \& Smith spectra.

${ }^{8}$ These LHB temperatures have not taken into account absorption within the LHB. Assuming an absorbing column $\sim 4 \times$ the temperatures drop by $\Delta \log T \sim 0.01$. 3

${ }^{\mathrm{h}}$ This paper assumed a second, hotter component with $\log T_{D 2} \sim 6.4$.

iThis temperature was derived in the anti-center. Snowden et al. (1990b) showed that the temperature of the LHB de galactic longitude; with galactic center directions having slightly higher temperatures than the galactic anti-center.

${ }^{k}$ Result obtained with the BeppoSAX LECS. The temperatures were derived using the Mewe-Kaastra-Liehdal code. T! should not be compared to the current work because the Parmar et al. (1999) data selection does not well sample the $g$ c

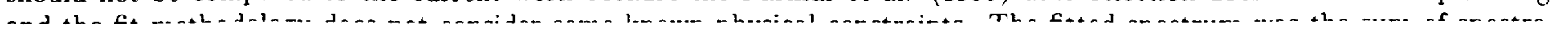




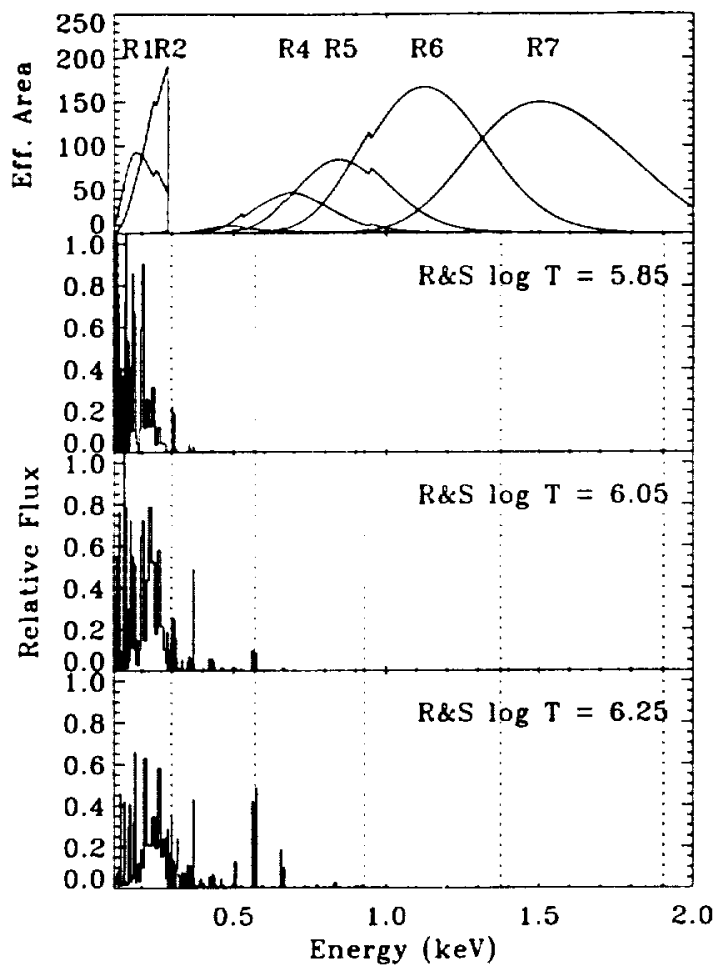

Fig. 1.- A comparison of the spectra of thermal components with the available independent spectral elements for the ROSAT PSPC. Top: the ROSAT PSPC band response functions. Below: Three thermal spectra spanning the range of temperatures found in the literature. The vertical lines show the divisions between independent spectral resolution elements, i.e., elements with nonoverlapping FWHM. The bulk of the emission from galactic thermal components falls almost entirely within two independent spectral resolution elements.

given some a priori model of the EPL, the four fit parameters necessary to describe two thermal components are under-determined. In practice, the situation is even worse. Figure 1 shows the Raymond \& Smith (1977) spectra for two representative thermal components, and a maximal set of independent resolution elements. (As with all future references to the Raymond \& Smith spectra in this paper, except in the context of XSPEC, we are using the 1991 release with cosmic abundances.) The bulk of the emission is contained in

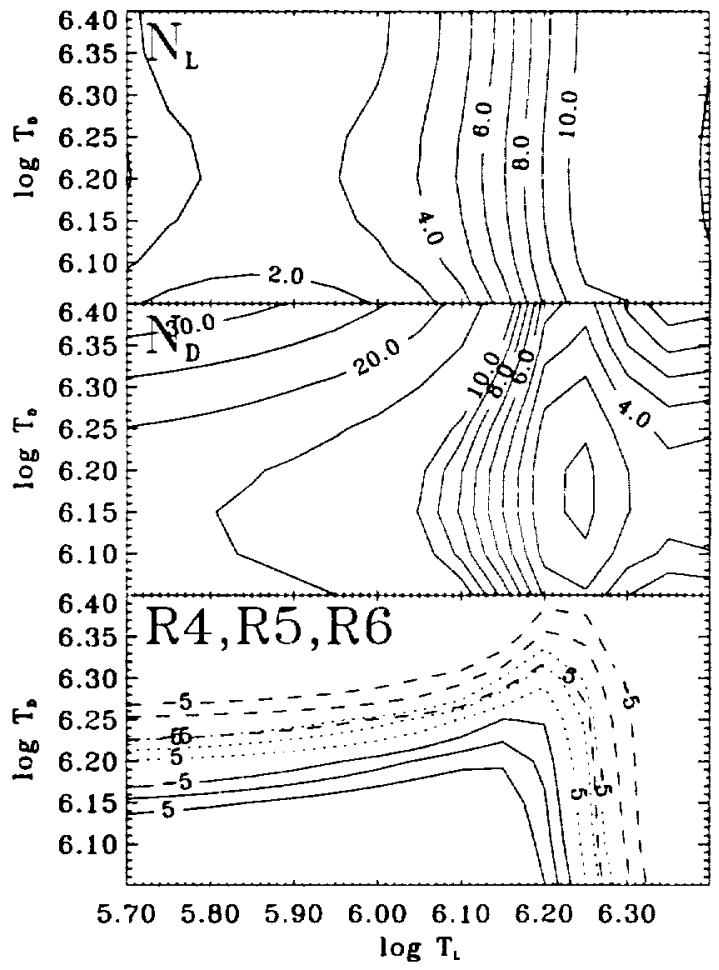

Fig. 2.- The degeneracy in the thermal component fit parameters. We have used a "mean" high galactic latitude background spectrum with a mean absorbing column $\left(N_{b}\right)$ of $1.21 \times 10^{20} \mathrm{~cm}^{-2}$ (and $N_{f}=0$ ), have assumed a Chen, Fabian \& Gendreau (1997) EPL (their Model A), and have assumed a single-temperature distant component. Top: The range of normalizations required for the local emission component, in units of $1000 n_{e} n_{p} L$ ( $n_{e}$ and $n_{h}$ in $\mathrm{cm}^{-3}$ and $L$ in $\mathrm{pc}$ ), as a function of the local and distant component temperatures. Middle: The same as above for the distant component. Bottom: The region of parameter space allowed by the R4, R5, and R6 band fluxes, allowing a $\pm 5 \times 10^{-6}$ counts $\mathrm{s}^{-1}$ arcmin $^{-2}$ uncertainty in the fluxes. Solid: R4, Dotted: R5, and Dashed: R6. Results are similar for other EPL models, though no EPL model can force the three regions to have significant overlap.

$\sim 2$ resolution elements.

At the risk of appearing to labour the point, consider the following. The six standard ROSAT PSPC bands R1-R7 (the R1 and R2 bands form- 
ing the $\frac{1}{1} \mathrm{keV}$ band, the R4 and R5 bands forming the $\frac{3}{4} \mathrm{keV}$ band, and excluding R3 as usual, Snowden et al. 1995) contain all of the information in the PSPC spectrum. The bulk of the information about the thermal components is contained in the $R 1$ and $R 2$ bands. Using only the $R 1$ and $R 2$ bands, given the absorbing column and the EPL, one can choose (within reason) any pair of local and distant temperatures, $T_{L}$ and $T_{D}$, and find some pair of normalizations $N_{L}$ and $N_{D}$ that provide the observed $R 1$ and $R 2$ band values. Figure 2 shows the ranges of normalizations required over a reasonable range of $T_{L}$ and $T_{D}{ }^{4}$.

Also shown in Figure 2 is the region allowed by the $\mathrm{R} 4, \mathrm{R} 5$, and $\mathrm{R} 6$ band values. The horizontal branch of the allowed parameter space represents a "minimal LHB" model, where the $\frac{1}{4} \mathrm{keV}$ flux is dominated by the distant emission; the LHB component is then poorly constrained and can assume a broad range of values. The vertical branch of the allowed parameter space represents a "maximal LHB" model, where the $\frac{1}{4} \mathrm{keV}$ flux is dominated by the LHB; the distant emission is then poorly constrained and can assume a broad range of values. It should also be noted that the vertical branch falls at the $T_{L}$ at which the R2/R1 ratio for the local component is a maximum (see Figure 3.)

Most of the published parameters of the local and distant thermal components fall in the region allowed by the R4 flux.

The "degeneracy" in the fit parameters can be broken with the addition of angular information; if $N_{b}$ varies over scales smaller than the angular scales of the variation in the emission, then the local and distant normalizations can be determined, and from them, the temperatures. It is not our desire to single out any particular practitioner of spectral fitting for criticism, especially since the thermal parameters were not their prime objective. However, one can easily show that the thermal components fit, for example, by Chen et al. (1997) are incorrect. Taking a small strip of the $R A S S$ in the southern galactic hemisphere that is free of strong $\frac{1}{4} \mathrm{keV}$ structures $\left(\ell=165^{\circ},-55^{\circ}>\right.$ $\left.b>-75^{\circ}\right)$, and assuming that the temperatures of the local and distant emission do not vary with

${ }^{4}$ The data used in the construction of Figure 2 were regions with $b>45^{\circ}, 45^{\circ}<\ell<270^{\circ}$, and $1.1 \times 10^{20}<N_{H}<$ $1.3 \times 10^{20} \mathrm{~cm}^{-2}$.

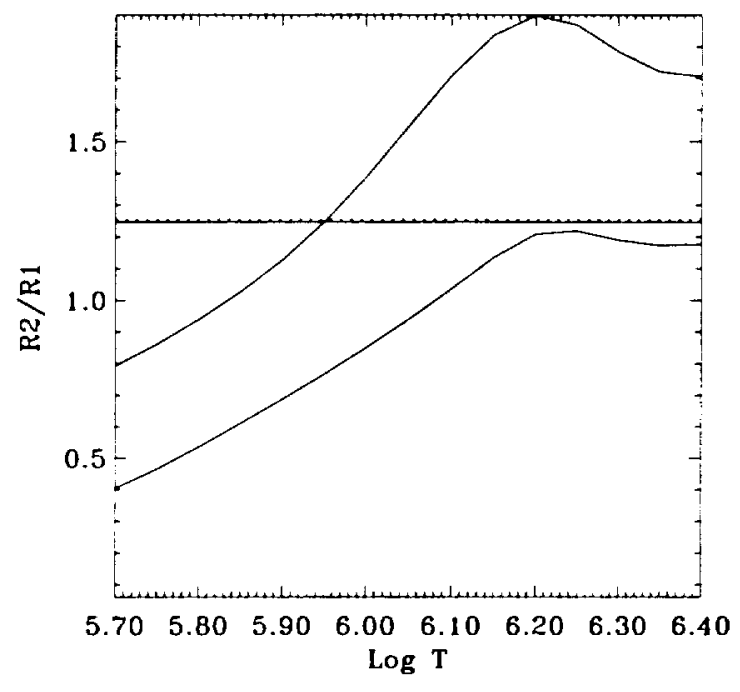

Fig. 3.- The $\mathrm{R} 2 / \mathrm{R} 1$ ratio as a function of temperature. The top curve is the $R 2 / R 1$ ratio for the distant component after absorption by a "mean" high galactic latitude column of $1.21 \times 10^{20} \mathrm{~cm}^{-2}$; the bottom curve is the ratio for the local component. The horizontal line is the ratio for the "mean" high galactic latitude background. Note that since the local component has an R2/R1 ratio less than the observed $R 2 / R 1$ ratio in all cases, the distant component must have a temperature greater than $\log T \sim 5.95$.

position, one can solve for the normalizations as a function of position. A plot of the normalization as a function of absorbing column density, Figure 4 , shows a strong correlation between the absorbing column density and the normalization of the distant component. One should not expect the emission from beyond the absorbing layer to be correlated with the intervening column density of $\mathrm{H}$ I. Therefore, the normalization must be wrong.

Indeed, repeating the exercise using the parameters determined by Snowden et al. (1998), shows no correlation between the absorbing column density and the normalization for the distant component. The uncertainties for the Snowden et al. (1998) parameters are larger than for the Chen et al. (1997) parameters because a softer distant component, absorbed by the intervening column, will require a greater change in $N_{D}$ before effecting the observed flux. 


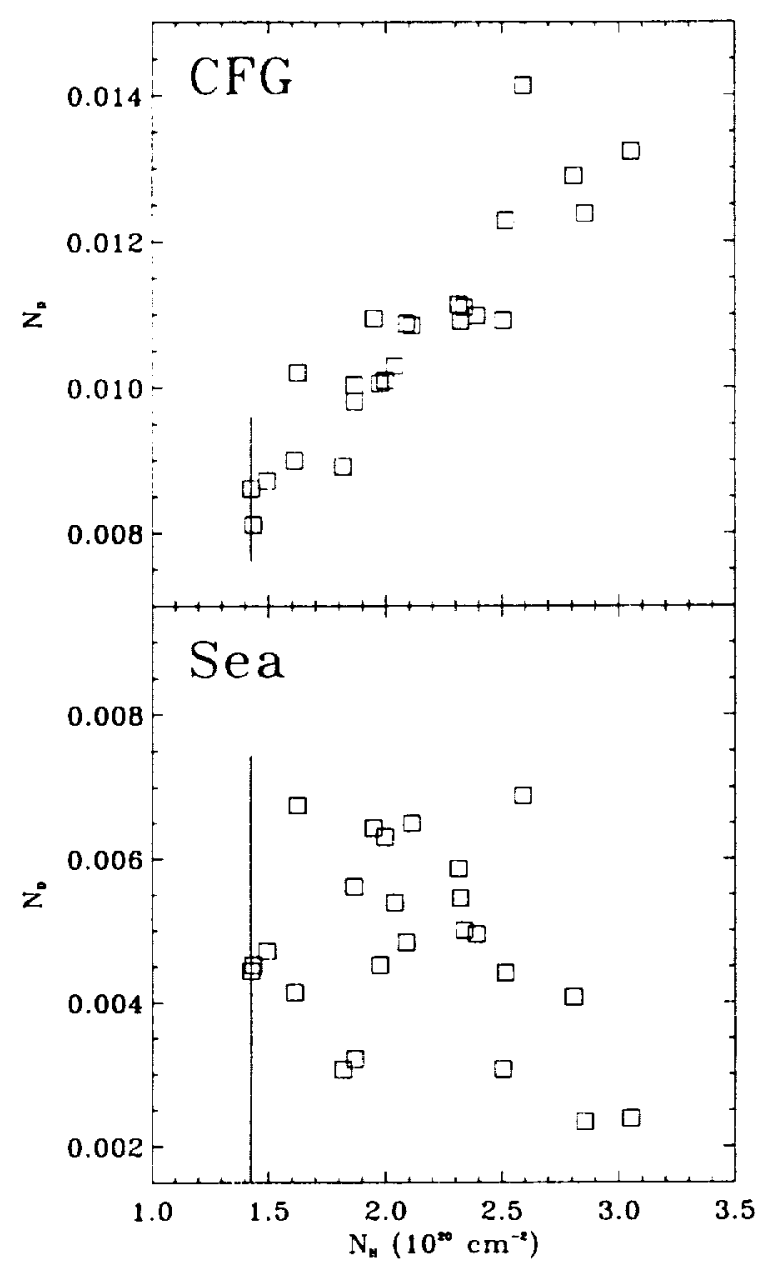

Fig. 4.- Checking spectral fit parameters using absorption. Top: We have used the temperatures of Chen, Fabian \& Gendreau (1997) $\left(\log T_{L}=5.76\right.$ and $\left.\log T_{D}=6.14\right)$ to demonstrate how the angular variation of the absorption can be used to check the temperature and normalizations derived from spectral fitting. Since one does not expect the true magnitude of the TAE to be correlated with the absorbing column, the fact that the derived TAE normalization is correlated with $N_{H}$ indicates that the assumed component temperatures are incorrect. Bottom: We have used the temperatures of Snowden et al. (1998) $\left(\log T_{L}=6.12\right.$ and $\log T_{D}=6.09$ ). There is no visible correlation between the TAE normalization and the absorbing column.
Consider a spatially invariant background absorbed by a spatially varying column of $\mathrm{H} \mathrm{l}$, to which is added a spatially invariant foreground. If one assumes that the background component has a higher temperature than the true background, then the assumed effective absorption cross-sections will be lower than the true effective absorption cross-sections. Thus the expected variation due to absorption (in the value of the transmitted flux) will be smaller than the true variation, and that part of the true variation that is not accounted for by the assumed variation will be attributed ( $b y$ whatever fitting mechanism one is using) to a variation in the strength of the background component. Thus, if the assumed temperature of the distant component is wrong, or if the magnitude of the distant component is wrong for any reason, the fitted normalization will be correlated with the absorbing column.

\subsection{All-Sky Deabsorption}

The second approach to determining the $\mathrm{X}$ ray background spectrum utilizes the galactic absorption as a tool to separate the local emission from the distant emission. One assumes that at some angular scale the foreground and background emissions are constant at some $\overline{I_{L}}$ and $\overline{I_{D}}$, and that the variation in observed X-ray flux is due to absorption by the intervening column. Given a tracer of the absorbing column (e.g., either an H I map or an IRAS $100 \mu \mathrm{m}$ map) one fits the relation in Equation 1 to find $\overline{I_{L}}$ and $\overline{I_{D}}$.

Since we have very little knowledge of the distribution of $N_{f}$, the absorption due to clouds within the LHB, one fits $\overline{I_{L}^{\prime}}$ and $\overline{I_{D}}$ in

$$
I_{O}-I_{E} e^{-\left(N_{b}\right) \sigma\left(\nu, N_{b}, S\right)}=I_{L}^{\prime}+I_{D} e^{-\left(N_{b}\right) \sigma\left(\nu, N_{b}, S\right)}
$$

where

$$
I_{L}^{\prime}=I_{L} e^{-\left(N_{f}\right) \sigma\left(\nu, N_{f}, \mathcal{S}\right)} .
$$

When using the $I_{L}^{\prime}$ determined from several bands to solve for the temperature and emission measure of the local component, a uniform $N_{f}$ is generally assumed (see $\S 4.2$ ). $N_{f}$ is small so, at worst, it can contribute a few percent error.

\subsection{1. $\frac{1}{4} \mathrm{keV}$ (The R12 Band)}

Snowden et al. (1998) used the single band RASS maps of Snowden et al. (1997) to separate 
the $\frac{1}{4} \mathrm{keV}$ (R12 band) emission into foreground and background components over the entire sky: Although these maps have a mean exposure much lower than pointed observations, they have the advantage of having the best possible LTE and SSX removal (Snowden et al. 1995), far better than can be accomplished for any single typical PSPC pointing.

Snowden et al. (1998) assumed that both the local emission and the distant emission were flat on $\sim 4^{\circ} .8$ scales, and they fit Equation 1 assuming $N_{f}=0$. The fit for each $4.8 \times 4.8$ region was then applied to center ninth of that region. They used the IRAS $100 \mu \mathrm{m}$ map of Schlegel et al. (1997) as a tracer of the absorbing column. This map was scaled by the mean value of $\mathrm{H} \mathrm{I} / I_{100}$ where the 21 cm data $\left(-74 \mathrm{~km} \mathrm{~s}^{-1}<V<24 \mathrm{~km} \mathrm{~s}^{-1}\right)$ was taken from the Dwingeloo survey of Hartmann \& Burton (1997). The EPL was assumed to have a $\Gamma$ of 1.96 (as per Hasinger et al. 1993) but was normalized to be consistent with the observed upper limit to the extragalactic background in the R12 band (Barber \& Warwick 1994; Snowden \& Pietsch 1995; Barber et al. 1996; Cui et al. 1996); although an inconsistent procedure, the inconsistency is inconsequential, as shown by Snowden et al. (2000). The decomposition of the emission into foreground and background components was done separately for the $R 1$ and $R 2$ bands, and the R2/R1 band ratio was then used to determine the temperatures of the local and distant components.

This method works well if the emission and absorption is well described by the canonical model. If, however, the emission and the absorption are interleaved, then significant overestimates of the distant component will occur. Conversely; if the absorption tracer underestimates the total absorbing column, then the distant emission will be underestimated. Both problems pertain to regions with high and intermediate velocity complexes. (See Herbstmeier et al. 1995 and Kerp et al. 1999 for preliminary studies of this problem.)

As can be seen in Snowden et al. (1998), Figure 8, (and again in our Figure 9) the distant emission appears to be strongly mottled, even away from the problematic high velocity clouds, and there is evidence of temperature variation. Surprisingly, none of the structure observed in the $\frac{1}{4} \mathrm{keV}$ TAE is seen in the $\frac{3}{4} \mathrm{keV}$ band, which is smooth (see Figure 4 in Snowden et al. (1997) or our Figure 9). This difference led Snowden et al. (1998) to conclude that the component responsible for the structure in the $\frac{1}{4} \mathrm{keV}$ band must not have significant emission in the $\frac{3}{4} \mathrm{keV}$ band, and must be different from the component producing the very smooth $\frac{3}{4} \mathrm{keV}$ emission. Accordingly, they felt justified in measuring the temperature of the component producing the $\frac{1}{4} \mathrm{keV}$ structure from the $\mathrm{R} 2 / \mathrm{R} 1$ band ratio alone, leaving the measure of the hotter component to groups concentrating on the $\frac{3}{4} \mathrm{keV}$ band.

Note that almost all other measures of the temperature of the TAE assumed a single-temperature component, and therefore measure a significantly higher temperature than Snowden et al. (1998). In this case, visible structure in the $\frac{1}{4} \mathrm{keV}$ (R12) band but relative smoothness in the $\frac{3}{4} \mathrm{keV}$ (R45) band is understandable; for $\log T=6.14, \mathrm{R} 45 / \mathrm{R} 12$ $\sim 0.026$, so much of the structure visible in the $\frac{1}{4} \mathrm{keV}$ band will be reduced to the level of the noise in the $\frac{3}{4} \mathrm{keV}$ band.

Since the effective cross-section for a given energy band depends upon both the column density of the absorbing gas and the spectral shape of the background emission, one must assume a spectral shape (or temperature) for the distant emission before separating the observed emission into local and distant components. After the local/distant separation one can take the band ratios of the distant component to determine a new spectral shape for the distant emission, and repeat the process.

Did the assumption of the temperature of the distant emission bias the subsequent measure of the temperature of the distant emission? For a range of local and distant temperatures, we assumed a fixed single-temperature distant component, and determined the normalizations required to produce the total observed $R 1$ and $R 2$ band emission. Using those temperatures and normalizations we then created vectors of flux vs. absorbing column for both bands. Using a method similar to that used in Snowden et al. (1998), those vectors were then fit to derive the local and distant emission values, assuming the crosssections appropriate for a distant component with $\log T_{D}=6.09$. We used the $\mathrm{R} 2 / \mathrm{R} 1$ band ratio from the resulting components to compute a "derived" temperature. Figure 5 shows the "derived" temperature for a range of true (or input) local and distant temperatures. One can see that for 
many temperatures, the Snowden et al. (1998) method (without iteration) could produce a biased measure of the temperatures. However, for temperatures near the assumed temperature, the bias is small and does not preclude finding the true temperature through iteration. For all reasonable cases, the biases are $\Delta \log T_{L}<0.02$ and $\Delta \log T_{D}<0.04$, and so are on the order of the systematics of the analysis (e.g., choice of plasma emission model).

\subsection{2. $\frac{3}{4} \mathrm{keV}$}

A large-scale deabsorption technique was used in Snowden et al. (1997) to demonstrate the existence of the X-ray emitting bulge with a temperature of $\log T=6.6$ which dominates the galactic center and the Loop I region, but does not affect most of the high galactic latitude sky.

Pietz et al. (1998) took an interesting, though Procrustean. approach to determining the TAE in the $\frac{3}{4} \mathrm{keV}$ band. They applied simultaneous spectral fits to three deep PSPC images from the same region of the sky, each with a different absorbing column, to determine the temperature of the local and distant components. This method minimizes the problems with inadequate non-cosmic background subtraction and avoids the degeneracy described in $\$ 2.1$.

They then used the fitted temperature for the TAE to fit a single-temperature hydrostatic equilibrium model of the galactic halo to the observed $\frac{3}{4} \mathrm{keV}$ distribution. Given 1) that the high galactic latitude $\frac{3}{4} \mathrm{keV}$ emission is smooth, 2) that one does not expect the canonical model to work well near the plane, where the classical "infill" prob$1 \mathrm{em}^{5}$ remains unsolved, 3 ) that the flattened halo model produces very little variation at high galactic latitude, and 4) that the flattened halo model has its greatest variation in the direction of galactic center where the multiple emission components are very confused, it is not surprising that the

\footnotetext{
${ }^{5}$ The "infill" problem is as follows: at $\frac{3}{4} \mathrm{keV}$ and $1.5 \mathrm{keV}$ the average emission level in the galactic plane is roughly the same as the average emission at high galactic latitude, despite the fact that one would expect galactic disk to "shadow" the extragalactic flux. See, for example, Sanders et al. (1983). Thus, there must be an additional galactic source of diffuse $\frac{3}{4} \mathrm{keV}$ and $1.5 \mathrm{keV}$ emission that almost exactly compensates for the emission that is absorbed by the galactic disk. However, that source of emission remains unkown
}

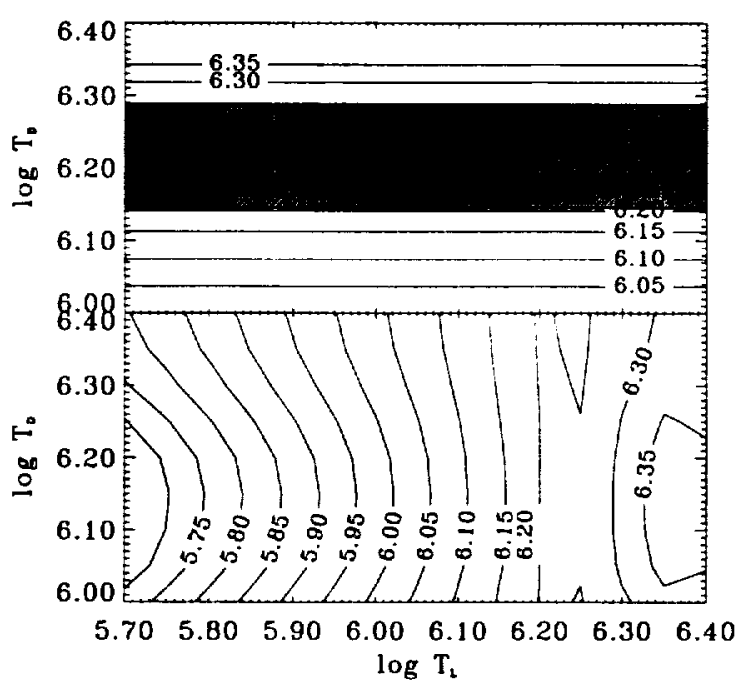

Fig. 5.- A demonstration of the bias due the method of Snowden et al. (1998). Input temperatures for both local and distant components were used to calculate a model of the observed flux as a function of absorbing column density. Those model data were then analyzed using the method of Snowden et al. (1998) to find "derived" temperatures for the local and distant components. The difference between the input and "derived" temperatures measures the bias in the Snowden et al. (1998) analysis. The axes are the input temperatures, the contours are the "derived" temperature. Top: The "derived" temperature of the distant component as a function of the true local and distant temperatures. The shaded region produces measured $R 2 / R 1$ ratios that do not correspond to any temperature. Bottom: The "derived" temperature of the local component as a function of the true local and distant temperatures. Making a bad initial assumption will produce an incorrect (biased) measure of the distant temperature, but iteration will produce the correct distant temeperature. 
Pietz et al. (1998) model is a "good fit" at $\frac{3}{4} \mathrm{kel}$. Their fitting has found the high galactic latitude normalization, but is not good at constraining the variation with latitude, since the region with the greatest lever arm is also the region that must be excluded due to galactic absorption and confusion with Loop I.

Pietz et al. (1998) used a very limited number of PSPC pointings towards a fairly atypical region (Draco) to make a local/distant separation at $\frac{1}{4} \mathrm{keV}$. They then claim that the distant component of the $\frac{1}{4} \mathrm{keV}$ flux (excluding regions with High Velocity Clouds or known structures such as Loop I) is produced solely by the extrapolation to $\frac{1}{4} \mathrm{keV}$ of the $\frac{3}{4} \mathrm{keV}$ emission produced by their galactic halo model. Their evaluation of the degree to which this extrapolation accounts for the angular structure of the $\frac{1}{4} \mathrm{keV}$ emission is overly optimistic; as they have no LHB model, they can always attribute poorly fitting regions to the effects of the LHB.

\subsubsection{Synthesis}

In Snowden et al. (2000), a large (but by no means comprehensive) catalogue of 378 ROSAT $\frac{1}{4} \mathrm{keV}$ band shadows ${ }^{6}$ was used to determine the values of the foreground and background emission in the R1 and R2 bands for many very localized regions at high galactic latitude for both the northern and southern galactic hemispheres. The $\frac{3}{4} \mathrm{keV}$ band emission was determined for the same regions by subtracting the absorbed EPL from the observed emission and deabsorbing the remainder. It was found that although both the distant $\frac{1}{4} \mathrm{keV}$ and $\frac{3}{4} \mathrm{keV}$ band emission varied by a factor of five, there was no clear correlation between the distant $\frac{1}{4} \mathrm{keV}$ emission and the distant $\frac{3}{4} \mathrm{keV}$ emission (see the Figure 15 from Snowden et al. 2000). The lack of correlation was used to support the argument made in Snowden et al. (1998) that the distant component that is responsible for the bulk of the $\frac{1}{4} \mathrm{keV}$ emission is not the same thermal component that is responsible for the $\frac{3}{4} \mathrm{keV}$ emission.

\footnotetext{
${ }^{6}$ An $X$-ray shadow is traditionally defined as a region that has a deficit of $X$-rays and the region of the deficit is spatially matched to an $\mathrm{H} \mathrm{I} 21 \mathrm{~cm}$ or $100 \mu \mathrm{m}$ emission feature. Snowden et al. (2000) expand that definition to regions with an excess of X-rays, where the excess is matched to the decrease in some measure of the absorbing column.
}

\subsection{Summary}

Spectral fitting methods are incapable of determining the parameters of the thermal components due to absorption uncertainty, poor non-cosmic background subtraction, and inadequate spectral resolution. The two large-scale deabsorption studies, executed in different bands, are each blind to the analysis in the other. Snowden et al. (1998) posited two components to the TAE, but for historical reasons deferred the necessary analysis in the $\frac{3}{4} \mathrm{keV}$ band to expand the proof of their case. Pietz et al. (1998) attempted to extrapolate their $\frac{3}{4} \mathrm{keV}$ results into the $\frac{1}{4} \mathrm{keV}$ band, but without taking into account the structure of the LHB, or the possibility of additional halo components.

The Snowden et al. (1998) self-consistent solution for the temperature of the distant component derives a low temperature distant component $(\log T \sim 6.07)^{7}$ that produces little $\frac{3}{4} \mathrm{keV}$ emission. Assuming that both the $\frac{1}{4} \mathrm{keV}$ and the $\frac{3}{4} \mathrm{keV}$ distant emission are produced by the same thermal component requires a higher temperature $(\log T>6.14)$ but, as shown in Figure 4 and $\S 2.1$, the higher temperature is inconsistent with the observed angular variation of the emission with absorbing column. Further, the extensive shadowing study by Snowden et al. (2000) shows that the distant $\frac{3}{4} \mathrm{keV}$ emission is not correlated with the distant $\frac{1}{4} \mathrm{keV}$ emission.

Therefore, the distant emission, the TAE, must have more than a single thermal component and, in fact, may have multiple thermal components. The temperature of those components may be spatially variable. The intensity is undoubtedly variable. Due to the limitations of the data, no more than an incremental improvement can be made by extending the Snowden et al. (1998) analysis to the R4-R7 bands. We will assume the TAE to be composed of two thermal components, a soft thermal component with temperature $T_{S}$ dominating the $\frac{1}{4} \mathrm{keV}$ band, and a hard thermal component with temperature $T_{H}$ dominating the $\frac{3}{4} \mathrm{keV}$ band. We will derive model temperatures for those components, and will evaluate the extent to which fur-

\footnotetext{
${ }^{7}$ The temperatures quoted here from Snowden et al. (1998) and Snowden et al. (2000) are not quite the temperatures found in those papers. The values here have been recalculated from the $\mathrm{R} 2 / \mathrm{R} 1$ band ratios using an updated detector response matrix, while the Snowden et al. papers used earlier versions to remain consistent with previous papers.
} 
ther thermal components may exist.

\section{Method}

Ideally; to determine the thermal and spatial structure of the TAE, one would use the angular structure of the absorbing column to separate the emission in each band into local and distant components, and then one would determine what combination of thermal components would produce the spectrum (or SED) of the distant emission. Unfortunately, the separation of the emission of any band into local and distant components requires knowledge of the spectrum of the distant component in order to calculate the appropriate effective cross-sections. Thus, any determination of the nature of the distant emission requires iterative solution of both the separation into local and distant components and the modeling of the thermal component structure. The extent to which such a process can be successful is strongly limited by the interplay of the response of the instrument, the spectrum of the emission, the energy dependence of the absorption cross-sections, and the available range of absorption column density.

In the following sections, we will briefly describe the available $\mathrm{X}$-ray data and some considerations concerning the measurement of the absorbing column. Section 3.2 describes the limitations to the separation of the X-ray data into local and distant components that were alluded to above. Section 3.3 describes the external constraints that can be used to set the non-TAE parameters in Equation 2. Finally, we detail the iterative method used to separate the local and distant components and to determine the thermal structure of the distant emission.

\subsection{Data}

\subsubsection{The RASS}

The X-ray data are the single-band $R A S S$ maps of Snowden et al. (1997), which have $12^{\prime} \times 12^{\prime}$ pixels. Of these band maps, the combined R1 and R2 bands (the R12 band) map forms a " $\frac{1}{4} \mathrm{keV}$ " map, the combined R4 and R5 bands (the R45 band) map forms a " $\frac{3}{4} \mathrm{keV}$ " map, and the combined $R 6$ and $R 7$ bands (the R67 band) map forms a "1.5 keV" map. Histograms of the statistical significance of the pixels in each band are given in

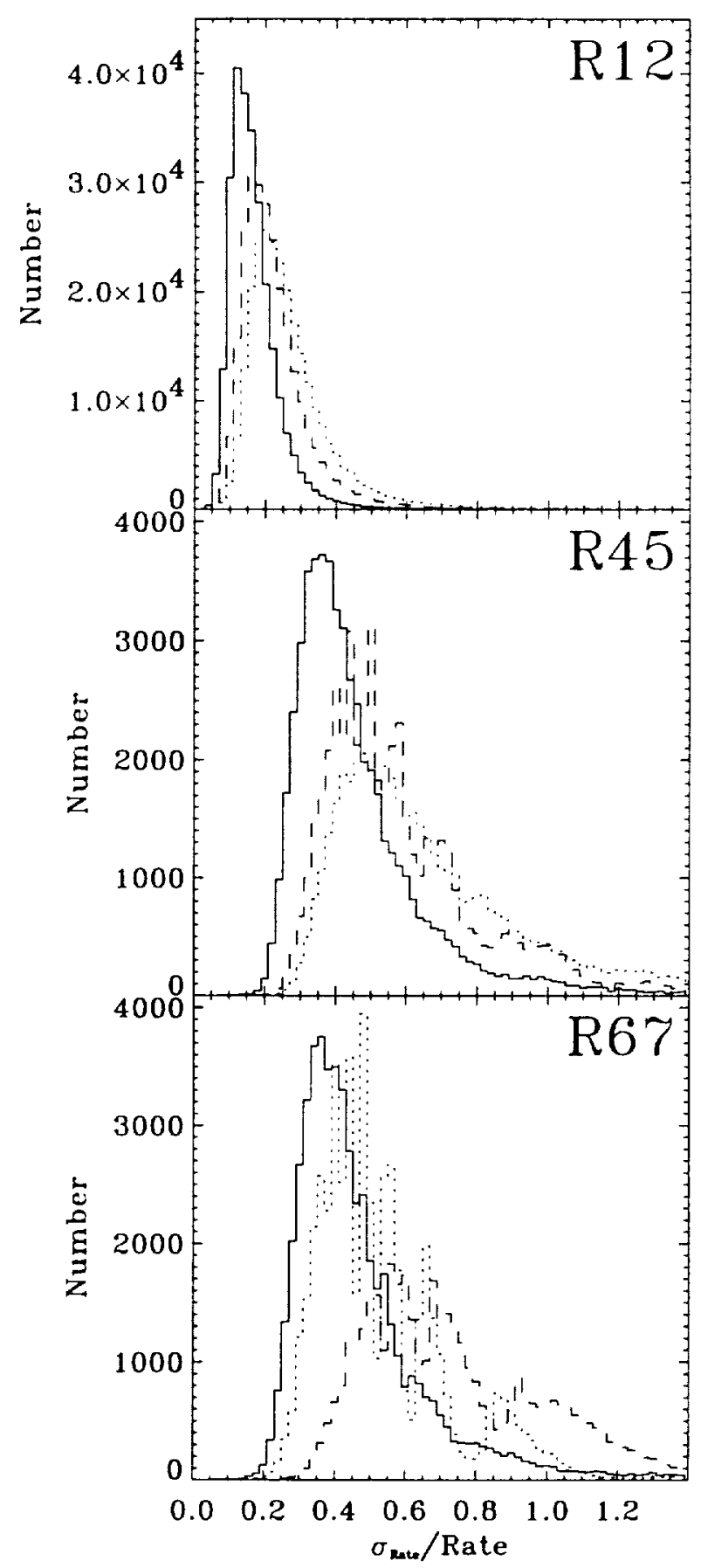

Fig. 6.- Histograms of the statistical significance of individual $12^{\prime} \times 12^{\prime}$ pixels in individual and combined bands of the RASS. For bands R4$\mathrm{R} 7$, only pixels in the north polar cap $\left(b>55^{\circ}\right.$, $45^{\circ}<\ell<270^{\circ}$ ) were used. In each panel, the solid line is the combined band, the dotted line is the lower energy band, and the dashed line is the higher energy band. 
Figure 6. Given the long temporal baseline possible with the RASS, the LTE and SSX removal were far better than is possible with any single pointed PSPC observation ${ }^{8}$. Some systematics remain. Even in seemingly uniform regions, a high contrast stretch of the raw $R A S S$ images will reveal the scan pattern, more strongly at $\frac{3}{4} \mathrm{keV}$ than at $\frac{1}{4} \mathrm{keV}$.

The R1 and R2 band maps have had point sources removed to a level of $(R 1+R 2)=0.025$ counts $\mathrm{s}^{-1}$ while the $\mathrm{R} 4-\mathrm{R} 7$ band maps have had point sources removed to a level of $(R 4+R 5)=(R 6+R 7)=0$. counts $\mathrm{s}^{-1}$. Correction of the RASS bands for the band-variable point source removal is discussed in $\S 3.3 .1$.

For reasons that will become apparent shortly, the analysis was restricted to $b>55^{\circ}, 45^{\circ}<\ell<$ $270^{\circ}$; the selection in galactic longitude was set to exclude the galactic center/Loop I emission. Within this region we also removed pixels contaminated by extended sources, Coma, Virgo, and A1367.

\subsubsection{The Absorbing Column}

We have taken as a tracer of the absorbing column the IRAS $100 \mu \mathrm{m}$ maps of Schlegel et al. (1997), as no other tracer of the absorbing column has either all-sky coverage, or angular resolution comparable to that of the RASS. As has been discussed in Arendt et al. (1998) $100 \mu \mathrm{m}$ emission traces, to varying degrees, the molecular, neutral atomic, and ionized atomic components of the ISM. (For a review see Chapters $3 \& 5$ of Kuntz 2000.)

Low velocity disk $\mathrm{H}$ I appears to be well traced by the $100 \mu \mathrm{m}$ emission, but the $100 \mu \mathrm{m}$ emissivity of the H I does appear to vary (Reach et al. 1998). The high and intermediate velocity $\mathrm{H} \mathrm{I}$ appears to have very low $100 \mu \mathrm{m}$ emissivity, and is essentially invisible at $100 \mu \mathrm{m}$, meaning that the IRAS 100 $\mu \mathrm{m}$ maps in regions with intermediate $(-100<$ $\left.v<-75 \mathrm{~km} \mathrm{~s}^{-1}\right)$ or high $\left(v<-100 \mathrm{~km} \mathrm{~s}^{-1}\right)$ velocity clouds will underestimate the absorbing column (Wakker \& Boulanger 1986).

${ }^{8}$ Removal of residual background contamination for individual PSPC pointings after reduction with the ESAS software (Snowden \& Kuntz 1998) can only be done by reference to the $R A S S$. The method is described by Kunt $z \&$ Snowden (1998).
Only upper limits to the $100 \mu \mathrm{m}$ emissivity of the $\mathrm{H}$ II have been determined, so the $100 \mu \mathrm{m}$ emission should be considered a poor tracer of the ionized gas (Arendt et al. 1998).

The emissivity of the molecular gas is a very strong function of the cloud temperature. Schlegel et al. (1997) attempted to correct for the cloud temperature in order to produce maps of the true dust column density, but the accuracy of this correction, denoted by $X$, is not well understood. Therefore the absorbing column of molecular gas Q Quen if one assumes a constant dust-to-hydrogennucleon ratio) may be poorly determined by the IRAS $100 \mu \mathrm{m}$ emission. However, molecular gas covers only a small portion of the high galactic latitude sky, appearing only where the $\mathrm{H} I$ column is greater than $3 \times 10^{20} \mathrm{~cm}^{-2}$ (Reach et al. 1998), and the $\mathrm{H}$ I $21 \mathrm{~cm} / \operatorname{IRAS} 100 \mu \mathrm{m}$ ratio is quite sensitive to the presence of molecular material.

The dominant tracer of absorption is $\mathrm{H} \mathrm{I}$. (At $\frac{1}{4} \mathrm{keV} \mathrm{He}$ provides roughly half of the physical absorption, while at energies $>0.5 \mathrm{keV}$ metals provide nearly all of the absorption.) The amount of absorption due to $\mathrm{H}$ II is a matter of debate (Arabadjis \& Bregman 1999; Laor et al. 1997); the cumulative absorbing column due to $\mathrm{H}$ II appears to be less than 0.2 that of the absorbing column due to $\mathrm{H} \mathrm{I}$ at $\frac{1}{4} \mathrm{keV}$ and may be significantly smaller.

Since the $100 \mu \mathrm{m}$ emission is very good at tracing the $\mathrm{HI}$, and the $\mathrm{HI} 21 \mathrm{~cm} / 100 \mu \mathrm{m}$ ratio is very sensitive to the presence of $\mathrm{H}_{2}$, the $100 \mu \mathrm{m}$ emission is the single best tracer of the total absorbing column. That the $100 \mu \mathrm{m}$ emission is not sensitive to $\mathrm{H} \mathrm{II}$ is offset by the $\mathrm{X}$-ray absorption being less sensitive to $\mathrm{H}$ II. To convert from the native units of dust emission $\left(\mathrm{MJy} \mathrm{sr}^{-1}\right)$ to absorbing column, we use the ratio of $N(\mathrm{H} \mathrm{I}) / X I_{100}$ determined from the northern galactic hemisphere $\left(b>45^{\circ}\right)$ where $N(\mathrm{H} \mathrm{I})<3 \times 10^{20} \mathrm{~cm}^{-2}$, a value with varies by $\sim 5 \%$, depending upon the latitude and column density limits used.

We have removed the regions where $N_{H}$ with $v<-75 \mathrm{~km} \mathrm{~s}^{-1}$ has column densities above $1 \times$ $10^{19} \mathrm{~cm}^{-2}$. This selection removes the strongest intermediate and high velocity clouds, which are known to be deficient in IRAS $100 \mu \mathrm{m}$ emission. 


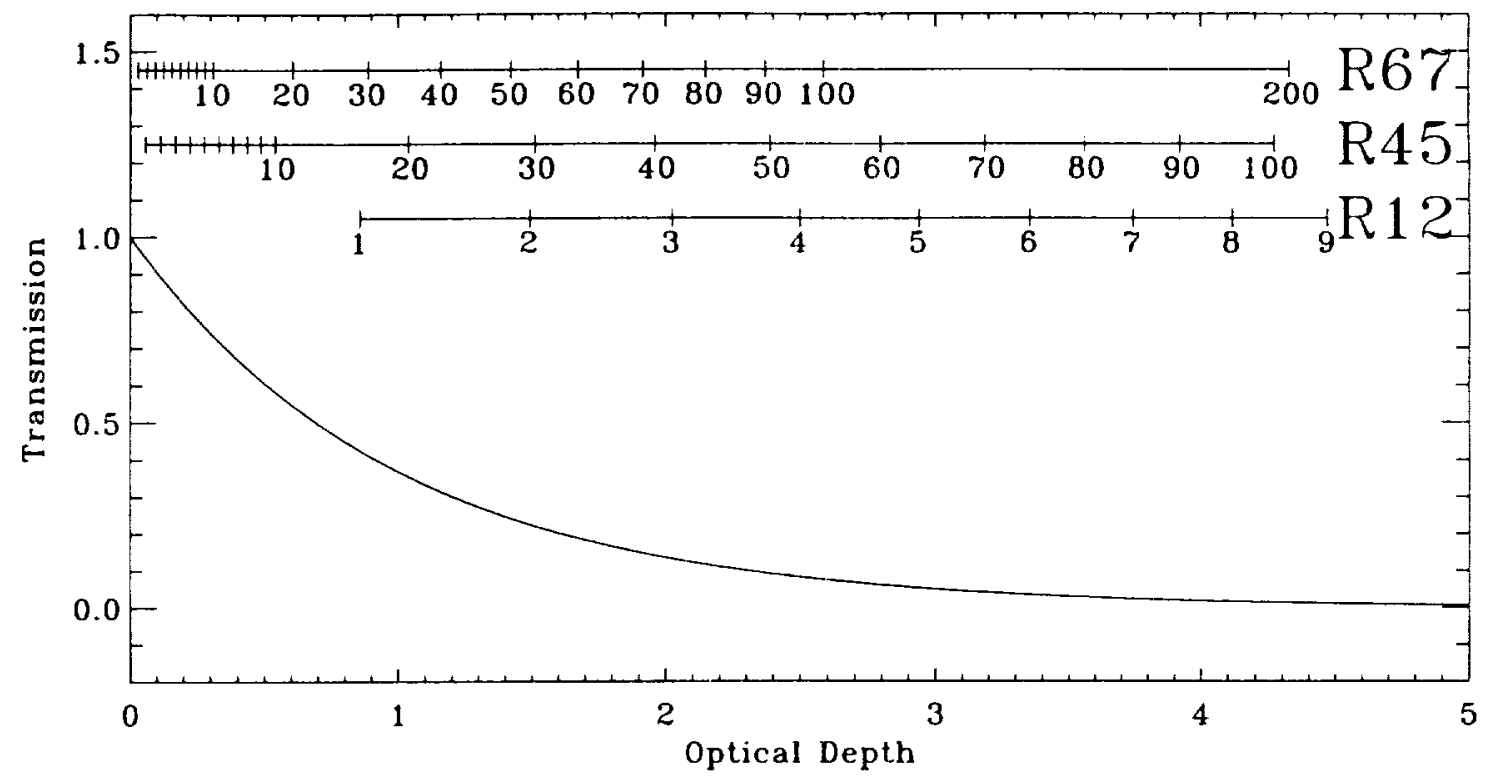

Fig. 7.- The column densities at various energies necessary to produce a given optical depth. The column density marked on each bar is in units of $10^{20} \mathrm{~cm}^{-2}$. We have assumed a $\log T=6.05$ thermal background in band $\mathrm{R} 12$, and $\log T=6.35$ thermal background for both $\mathrm{R} 45$ and R6 $\mathrm{T}$.

\subsection{Limits}

Optical Depths: In order to separate the distant emission from the local emission, one must have absorption over a wide range of optical depths within a limited angular region. (The region must be small in order for the assumption of a spatially invariant local and distant $\mathrm{X}$-ray emission made by Equation 1 to be valid.) In practice, the smallest $\tau$ must be $\lesssim 1$, in order to produce a good measure of $I_{D}$, the distant emission, and the largest $\tau$ must be $\gtrsim 2$ in order to produce a good measure of $I_{L}$, the local emission. Either of these limits can be relaxed if the other end of the optical depth range extends well beyond its limit. If the first condition is not met, but the latter is, $I_{D}$ may be estimated from $I_{D}=\left(I_{O}-I_{L}\right) e^{\tau}$, where $I_{O}$ is the observed flux.

Since the absoption cross section, $\sigma \propto E^{-8 / 3}$, regions fulfilling the $\tau$ requirements at $\frac{1}{4} \mathrm{keV}$ generally do not fulfill the $\tau$ requirements at $\frac{3}{4} \mathrm{keV}$ or $1.5 \mathrm{keV}$ (see Figure 7). Separation at $\frac{1}{4} \mathrm{keV}$ is usually possible if $\overline{N_{H}}<4 \times 10^{20} \mathrm{~cm}^{-2}$, whereas separation at $\frac{3}{4} \mathrm{keV}$ is generally only possible if $\overline{N_{H}} \gg 4 \times 10^{20} \mathrm{~cm}^{-2}$. Thus, while $\frac{1}{4} \mathrm{keV}$ sepa- ration into $I_{D}$ and $I_{L}$ is usually possible at high Galactic latitude, $\frac{3}{4} \mathrm{keV}$ separation is generally only possible at low latitude.

Count Rates: The difficulty in separating $I_{D}$ from $I_{L}$ at $\frac{3}{4} \mathrm{keV}$ is exacerbated by the $\frac{3}{4} \mathrm{keV}$ count rates, which are typically one-tenth of the count rates at $\frac{1}{4} \mathrm{keV}$ (note the lower statistical significance of higher energy band pixels in Figure 6). Thus, separation of the RASS is generally possible for the R1 and R2 bands, individually, but at higher energy, it is only possible in the combined $\mathrm{R} 45$ and $\mathrm{R} 67$ bands.

Regions with sufficiently high column densities to show good $\frac{3}{4} \mathrm{keV}$ shadows are typically near the galactic plane, where there are likely to be many emission components (and absorbing screens) along a given line of sight. Thus, although there are regions for which the local/distant separation is mathematically possible, those regions are unlikely to produce a meaningful measure of either the local or the distant emission. 


\subsection{External Constraints}

\subsubsection{Properties of the EPL}

The EPL is the aggregate of unresolved sources. It must be the result of a complex convolution of source populations with different spectra, the distribution of redshifts, and the distribution of intrinsic absorption. None of the existing populations of point sources thought to compose the EPL has spectra as hard as that of the EPL. Given the cosmological significance of the EPL, a number of groups have attempted to measure the photon index $(\Gamma)$ and normalization $\left(N_{\Gamma}\right)$ of the EPL (i.e., the spectrum of the aggregate of unresolved sources). Indices measured from ROSAT data alone have been higher than those measured from $A S C A$, which provides a significantly longer, absorption free, lever arm (e.g. Chen et al. 1997). It has been suggested by a number of authors that this discrepancy is due to calibration uncertainties in the ROSAT PSPC. Fits using both ROSAT and $A S C A$ data tend to indices more similar to the $A S C A$ values. We have chosen the values of Chen et al. (1997), $\Gamma=1.46, N_{\Gamma}=10.5 \mathrm{keV}$ $\mathrm{cm}^{-2} \mathrm{~s}^{-1} \mathrm{sr}^{-1} \mathrm{keV}^{-1}$, as being representative of a number of recent measures of the EPL from joint ROSAT/ASCA fits.

The Chen et al. (1997) point source removal limit for the region from which the $\Gamma=1.46$, $N_{\Gamma}=10.5$ EPL was derived was $\sim 0.00524$ counts $\mathrm{s}^{-1}$ for the R47 band. To correct to the point source detection limit of the RASS, nearly an order of magnitude larger, we used the $\log N-\log S$ relation of Hasinger et al. (1998) and have assumed a photon index for the point sources between the point source removal limits of Chen et al. (1997) and the RASS. We have assumed that those point sources can be characterized by the point sources detected by Chen et al. (1997) at somewhat lower fluxes $\left(\leqslant 0.00524\right.$ counts $\mathrm{s}^{-1}$ but $\gtrsim 0.0003$ counts $\left.\mathrm{s}^{-1}\right) . \mathrm{A} \Gamma=2.54$ index was derived by Chen et al. (1997) for a sample of confirmed QSO's detected in this flux interval. However, the confirmed QSO sample is softer than the complete sample of objects in this flux interval, so a harder index may be more apropriate. We have performed the analysis using both $\Gamma=2.54$ and $\Gamma=1.46$ for the point source correction ${ }^{9}$.

${ }^{9}$ The point source correction for $\Gamma=2.54$ requires a nor-
Caveat: Although much effort has been expended to determine the spectra of individual sources, the spectrum of the unresolved extragalactic background, the EPL, is unknown in the $\frac{1}{4} \mathrm{keV}$ band. We have assumed that the power law that describes the extragalactic background for $E>1.0 \mathrm{keV}$ extends into the $\frac{1}{4} \mathrm{keV}$ band.

It has been suggested that the spectrum of the extragalactic background turns up below $1 \mathrm{keV}$. However, that turn-up can not be too great; Snowden et al. (2000) show that the isotropic extragalactic background at $\frac{1}{4} \mathrm{keV}$ cannot be more than $\sim 325 \times 10^{-6}$ counts s${ }^{-1}$ arcmin $^{-2}$. If the spectrum breaks at $1 \mathrm{keV}$, then $\Gamma<1.64$; if it breaks at $0.75 \mathrm{keV}$, then $\Gamma<1.67$. Thus, even if there is a turn-up in the extragalactic spectrum, the effect is small.

\subsection{2. $\frac{3}{4}$ keV Emission from the $L H B$}

Given all of the caveats placed on $\frac{3}{4} \mathrm{keV}$ lo$\mathrm{cal} /$ distant separation in $\S 3.2$, any external constraint on the magnitude of the local $\frac{3}{4} \mathrm{keV}$ emission is useful. Lines of sight useful for measuring the local component of the $\frac{3}{4} \mathrm{keV}$ emission must be chosen carefully. The highest signal-tonoise measure of the local $\frac{3}{4} \mathrm{keV}$ emission remains that of Snowden et al. (1993) towards MBM 12, (a molecular cloud situated just inside the LHB) which yields $2 \sigma$ upper limits of $23 \times 10^{-6}$ counts $\mathrm{s}^{-1} \operatorname{arcmin}^{-2}\left(\frac{3}{4} \mathrm{keV}\right)$ and $39 \times 10^{-6}$ counts $\mathrm{s}^{-1}$ $\operatorname{arcmin}^{-2}(1.5 \mathrm{keV})$. In this direction, the $\frac{1}{4} \mathrm{keV}$ flux is $385 \times 10^{-6}$ counts s $\mathrm{s}^{-1}$ arcmin $^{-2}$.

If the temperature of the LHB is $\sim \log T_{L}=$ 6.10 , then the $R 45 / R 12$ ratio is 0.017 , and the $\frac{3}{4} \mathrm{keV}$ count rate due to the LHB in the direction of MBM 12 is $7.1 \times 10^{-6}$ counts $\mathrm{s}^{-1} \operatorname{arcmin}^{-2}$, well below the upper limit and similar to the measured value of $\sim 5 \times 10^{-6}$ counts $\mathrm{s}^{-1} \mathrm{arcmin}^{-2}$. At high galactic latitudes, where the LHB flux is on the order of $400-600 \times 10^{-6}$ counts $\mathrm{s}^{-1} \operatorname{arcmin}^{-2}$, the $\frac{3}{4} \mathrm{keV}$ counts due to the LHB will be $8.75-13.25 \times$ $10^{-6}$ counts s$^{-1}$ arcmin $^{-2}$, which is a substantial

malization of $8.032 \times 10^{-13} \mathrm{erg} \mathrm{cm}^{-2} \mathrm{~s}^{-1} \mathrm{deg}^{-1}$ in band $\mathrm{R} 47$ and produces the corrections $\mathrm{R} 1=-45.9, \mathrm{R} 2=-38.7$, $\mathrm{R} 4=4.3, \mathrm{R} 5=5.1, \mathrm{R} 6=6.6, \mathrm{R} 7=3.2$, where the values are in units of $10^{-6}$ counts $\mathrm{s}^{-1} \mathrm{arcmin}^{-2}$ to be added to the EPL. The point source correction for $\Gamma=1.46$ requires a normalization of $9.712 \times 10^{-13} \mathrm{erg} \mathrm{cm}^{-2} \mathrm{~s}^{-1} \mathrm{deg}^{-1}$ in band $\mathrm{R} 47$ and produces $\mathrm{R} 1=-14.3, \mathrm{R} 2=-15.3, \mathrm{R} 4=3.2$, $\mathrm{R} 5=4.9, \mathrm{R} 6=8.4, \mathrm{R} 7=5.9$. 
fraction of the high galactic latitude $\frac{3}{4} \mathrm{keV}$ flux, typically $\sim 35 \times 10^{-6}$ counts $\mathrm{s}^{-1} \operatorname{arcmin}^{-2}$. Thus, ignoring the LHB contribution at $\frac{3}{4} \mathrm{keV}$ in the manner of Pietz et al. (1998) is unwise.

\subsection{The Iterative Algorithm}

We use a bicyclic iterative scheme to solve for the distant component in each band and, simultaneously, fit two thermal components to the resulting SED. The process is described graphically in Figure 8. Given the difficulty of performing a local/distant separation in the R4-R7 bands, we have treated those bands somewhat differently than the R1 and R2 bands; the R1 and R2 bands are separated into local and distant components with a method similar to that used by Snowden et al. (1998), while the R4-R7 bands are corrected only for galactic absorption after the contribution of the LHB has been removed. The lack of correlation between the $\frac{1}{4} \mathrm{keV}$ and $\frac{3}{4} \mathrm{keV}$ bands, as well as the low model temperature found for the $\frac{1}{4} \mathrm{keV}$ emission by Snowden et al. (1998) suggest that there is little contribution from the $\frac{1}{4} \mathrm{keV}$ emission to the $\frac{3}{4} \mathrm{keV}$ emission (when compared to the much greater flux at $\frac{1}{4} \mathrm{keV}$ ) and vice versa. The fitting algorithm begins with this assumption, but the assumption is not reified by the fitting algorithm.

The first cycle (the left side of Figure 8) iteratively determines the temperature of the softer component from the bands R1 and R2, after separating the local from the distant emission. The second cycle (the right side of Figure 8 ) iteratively determines the temperature of the harder component from the bands R4 through R7. Each cycle passes to the other the extrapolation into the complementary band range of the component it has fit. The first cycle finds the single best thermal component fit to the R1 and R2 bands, and extrapolates that component into the R4-R7 bands. That extrapolation is then subtracted from the $R 4-R 7$ data, and a single thermal component is fit to the remainder. That thermal component is then extrapolated to the R1-R2 band range, where it is subtracted from the R1-R2 data. The process is then repeated.

\subsubsection{Details of Local/Distant Separation}

The process by which the distant emission is separated from the local emission in bands $\mathrm{R} 1$ and R2 is similar to that used by Snowden et al. (1998). Both the local and distant emissions are assumed to be spatially invariant over $4^{\circ} .8 \times 4^{\circ} .8$ regions. The bulk of the distant emission in the R12 band is assumed to come from a single thermal component. From each RASS single band image is subtracted the contribution of the EPL, after absorption by the galactic ISM, and the contribution to that band from the hard thermal component, also after absorption by the galactic ISM. The X-ray data from each $4: 8 \times 4^{\circ} .8$ region are then binned by the absorbing column density into $1 \times 10^{19} \mathrm{~cm}^{-2}$ wide bins. The binned data are then fit by Equation 2, assuming some $\log T$ for the distant emission. The Lampton et al. (1976) criteria are used to determine the uncertainties in the fit parameters. The fitting is done for each $4.8 \times 4.8$ region for which the $\overline{N_{H}}<4 \times 10^{20} \mathrm{~cm}^{-2}$. The $4.8 \times 4.8$ regions overlap by $1^{\circ} .6$, so the solutions are applied only to the center ninth of the region $\left(1^{\circ} \cdot 6^{2}\right)$.

In their work, Snowden et al. (1998) found that the uncertainty in the distant component could be substantially reduced by taking the quantity

$$
I_{D}^{\prime}=\left(I_{O}-\bar{I}_{L}\right) e^{r}
$$

rather than the value produced by the fit. We have followed this procedure, although, owing to slight differences in the fitting procedure, we find that the improvement is less significant.

Once the separation is accomplished for both the $\mathrm{R} 1$ and the $\mathrm{R} 2$ band images, the hardness ratio is determined for the distant component. This leads to a new estimation of the $\log T_{S}$ for the distant emission, and the process is repeated until the assumed temperature and the temperature recovered from the hardness ratio agree to $\Delta \log T_{S}<0.005$. Convergence typically occurs in three iterations.

\subsubsection{Details of the R4-R7 SED Fitting}

From each RASS single band image is subtracted the contribution from the EPL, absorbed by the galactic ISM, the contribution from the soft thermal component that dominates the R1 and R2 bands, absorbed by the galactic ISM, and the contribution by the LHB, without absorption. The re- 


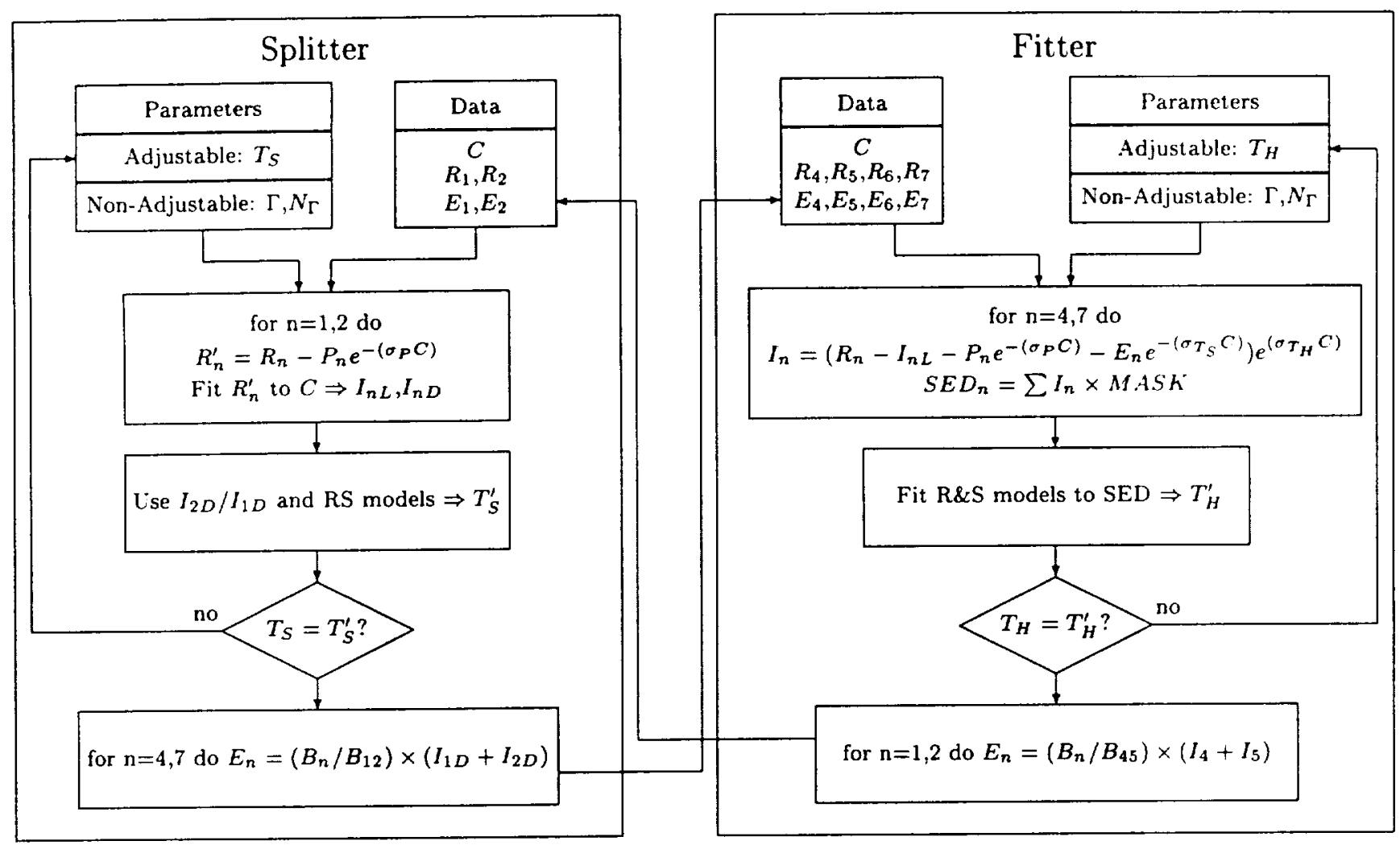

Fig. 8.- Flowchart for the bicyclic iterative fitting of two thermal components to the SED of the TAE. $T_{S}$ is the model temperature of the softer component (which is fit in the left iterative cycle) and $T_{H}$ is the model temperature of the harder component (which is fit in the right iterative cycle). $R_{n}$ is an image of the observed emission in band $n, E_{n}$ is an image of the emission extrapolated from other energies into band $n, I_{n}$ is an image of the TAE emission in band $n$, corrected for absorption, $P_{n}$ is the value of the extragalactic power law in band $n$, and $C$ is the absorbing column. The subscripts $L$ and $D$ denote the local and distant components. $B_{n}$ is the band value derived from a Raymond \& Smith spectrum. The " $T=T^{\prime}$ ?" is a consistency check for the temperature of the component.

mainder is smoothed by a three pixel boxcar and is then deabsorbed (i.e., multiplied by $e^{\tau}$ where $\tau$ is the effective cross-section multiplied by the column density) assuming some $\log T_{H}$ for the distant emission.

After all four bands have been deabsorbed in this manner, the mean deabsorbed SED for a region with $b>55^{\circ}$ and $45^{\circ}<\ell<270^{\circ}$ is calculated. A thermal model is fitted to this SED to determine the new $\log T_{H}$, and the process is repeated until $\Delta \log T_{H}<0.005$. Convergence typically occurs in three iterations. Convergence between the two halves of the iteration usually occurs in three or four iterations.

\section{Analysis of Current Results}

The results of the local/distant separation and the SED fitting are shown in Figure 9. The top images show the soft and hard components in the bands in which they dominate, and below that are their uncertainties ( $3 \sigma$ for the soft, $1 \sigma$ for the hard). The third row contains the spatial distribution of the temperature of the two components. For the soft band the temperature is in the form of the R2/R1 band ratio, while for the hard component the temperature is the fitted Raymond \& Smith (1977) equilibrium temperature. The fourth row contains the absorbing column, and the raw $\mathrm{R} 47$ band image. 


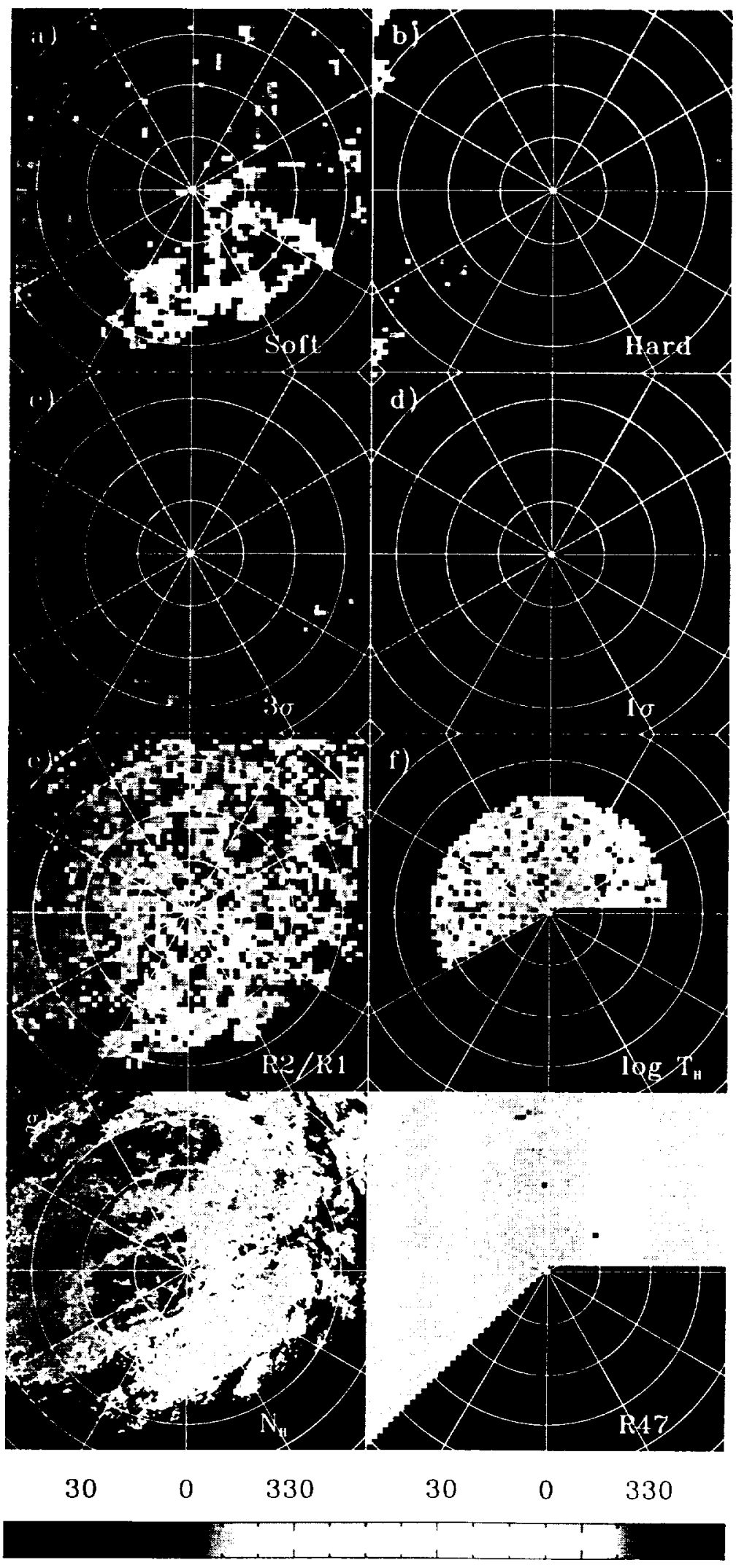


Fig. 9.- Previous page: The spatial distribution of measured quantities. All images are of the northern galactic hemisphere. Galactic longitude $0^{\circ}$ is down, $90^{\circ}$ is on the left. The longitude spacing is $30^{\circ}$, the latitude spacing is $15^{\circ}$. Note that the pixelization is 1.6 a a: The soft TAE component as seen in the R12 band. Greyscale: minimum 0 , maximum $5 \times 10^{-3}$ counts s $\mathrm{s}^{-1} \operatorname{arcmin}^{-2}$. $b$ : The hard TAE component as seen in the R47 band. Greyscale: minimum 0 , maximum $5 \times 10^{-4}$ counts s$^{-1} \operatorname{arcmin}^{-2}$. $c$ : The $3 \sigma$ uncertainty of the soft TAE component. Greyscale as in $a$. $d$ : The $1 \sigma$ uncertainty of the hard TAE component. Greyscale as in $b . e$ : The R2/R1 band ratio for the soft component. Greyscale: minimum 0, maximum 3. $f$ : The $\log$ of the fitted temperature of the hard TAE component. Greyscale: minimum 6.0, maximum 7.0. $g$ : The absorbing column density. Greyscale: minimum 0 , maximum $4 \times 10^{20} \mathrm{~cm}^{-2}$. $h$ : The observed R47 band flux. Greyscale as in $b$. Note that the bulk of the observed structure is due to the scan paths.

\subsection{The TAE}

\subsubsection{The Soft Component}

The temperature of the soft component was derived from the $\mathrm{R} 2 / \mathrm{R} 1$ ratio over the north polar cap region $\left(b>45^{\circ}, 45^{\circ}<\ell<270^{\circ}\right.$, excluding regions with strong $\mathrm{X}$-ray clusters of galaxies or strong $\left(N_{H}>10^{19} \mathrm{~cm}^{-2}\right)$ high and intermediate velocity clouds). The histogram of the $\mathrm{R} 2 / \mathrm{R} 1$ band ratio over the north polar cap region is shown in Figure 10, along with the conversion to Raymond \& Smith model temperatures. The FWHM of the $R 2 / R 1$ ratio is $0.670-1.190$, and the distribution is asymmetric, with a strong high $R 2 / R 1$ tail. The values in the high tail do not correspond to a Raymond \& Smith model temperature, and thus indicate that the bulk of the dispersion is due not to an intrinsic spread of temperatures, but to the uncertainty of the local/distant split. The mean uncertainty in $R 2 / R 1$, obtained by propagating the uncertainties in the distant components of $\mathrm{R} 2$ and $R 1$ is sufficient to produce the entire observed FWHM. Unlike Snowden et al. (1998), we have not smoothed the distant emission images before calculating the $R 2 / R 1$ ratios, so our uncertainties are somewhat higher.

In order to minimize the effects of the tail we have chosen to characterize the $R 2 / R 1$ ratio by the mode. The mode is 0.950 ; the corresponding model temperature is $\log T_{S}=6.056$. Snowden et al. (1998) found $\log T=6.09$ (FWHM $=$ $6.01-6.17$ ) for the soft distant component, without an extrapolation of a hard thermal component. If we follow Snowden et al. (1998), and ignore the extrapolation of the hard component, we find an R2/R1 ratio of 0.92 , with a corresponding model temperature of $\log T=6.040$. Since we used a much more restricted portion of the sky; and a different fitting algorithm, the difference between the value from Snowden et al. (1998) and our value (without the extrapolation of the hard component) is a good measure of the systematic uncertainties.

Systematics: Most of the systematics in the local/distant separation have been discussed in Snowden et al. (1998). The magnitude of the distant component is not correlated with the absorbing column, though the uncertainty will be. The value of the R2/R1 ratio for the distant component is not correlated with the value of $R 2, R 1$, or the magnitude of the absorbing column. Snowden et al. (2000) discuss the variation of the R2/R1 ratio of the distant component with galactic latitude and longitude (they find none), and although we have a greater number of pixels, Snowden et al. (2000) have the advantage because their regions were larger, and were carefully chosen to have a large dynamic range in absorption

Although the R2/R1 ratio of the distant component is not correlated with $N_{H}$, the derived temperature of the distant component may still be systematically affected by the absorbing column. If the absorbing column is systematically underestimated, one might overestimate the model temperature, and that overestimate may depend upon the absorbing column. As can be seen in Figure 11, for the approximate background temperature and range of columns used $\left(N_{H}<4.0 \times\right.$ $10^{20} \mathrm{~cm}^{-2}$ ), the overestimate has little dependence on absorbing column. If $N_{H I J} / N_{H I} \sim 0.33$ (Reynolds 1991) and $\sigma_{H I} / \sigma_{H I} \sim 0.62$ (assuming that $N_{H e I I} / N_{H e} \sim 0.5$ as per Domgörgen $\&$ Mathis 1994) then the absorbing column due to $\mathrm{H}$ II will be $\sim 0.2$ times that due to $\mathrm{H} \mathrm{I}$. Assuming a distant component temperature of $\log T=6.15$, if one does not account for the $20 \%$ 
extra absorption due to the H II, one would overestimate the distant component temperature by $\Delta \log T \sim 0.05$.

One might also expect to find that the lack of a sufficient dynamic range in the absorption

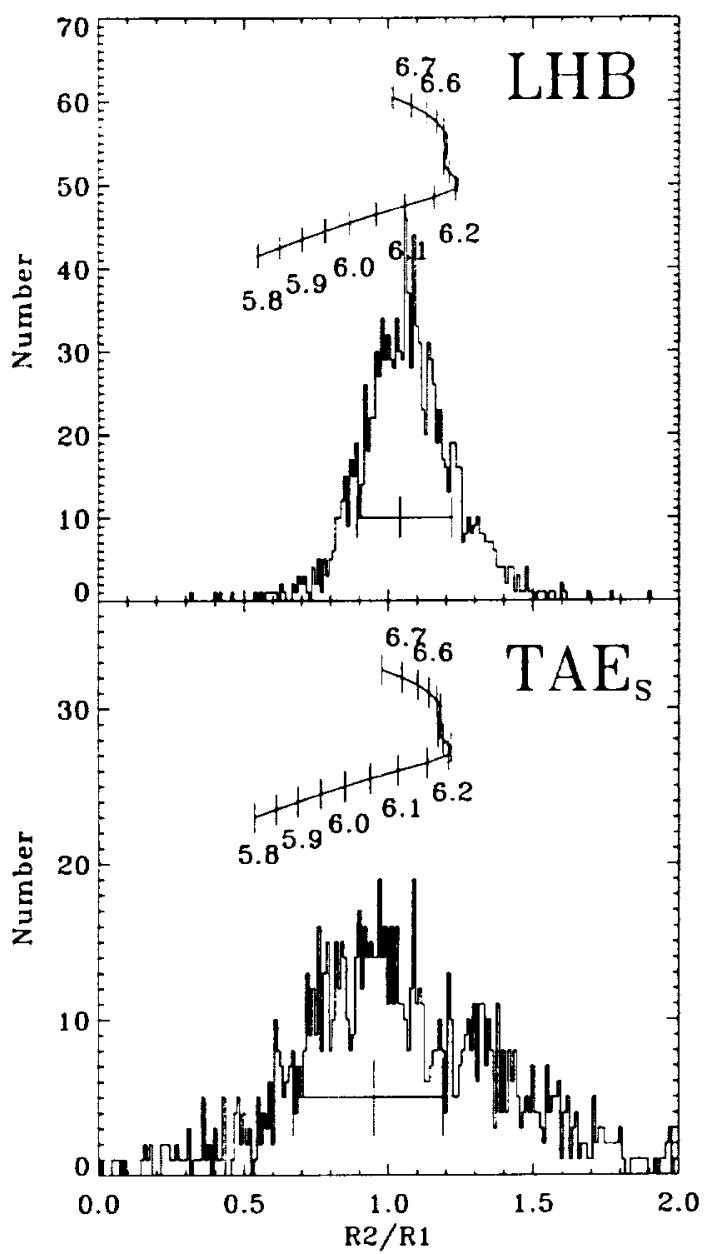

Fig. 10.- The distribution of the R2/R1 band ratios for the LHB and TAE. The squiggle above the histogram shows the conversion to Raymond \& Smith model temperature. For the TAE, both the $\mathrm{R} 2 / \mathrm{R} 1$ ratio and Raymond \& Smith conversion are after deabsorption. For the LHB the histogram is of the $\mathrm{R} 2 / \mathrm{R} 1$ ratio derived from $I_{L}^{\prime}$ values; the temperature conversion assumes a $4 \times 10^{18} \mathrm{~cm}^{-2}$ absorption. Note that only the lower branch of the temperature conversion is of interest as the upper branch would over-predict the R4 7 flux. might produce a systematically high (or low) estimate of the distant component. We recalculated the $\mathrm{R} 2 / \mathrm{R} 1$ ratio using only those $1: 6^{2}$ pixels with "good" fits, defining "good" fits to be those for which the $1 \sigma$ lower limit was greater than zero. The difference between this value of $R 2 / R 1$ and that calculated from all of the pixels was insignificant.

\subsubsection{The Hard Component}

The temperature of the hard component was derived from fitting the R4-R7 SED. The uncertainty of the value was determined from the dispersion of the temperatures found from fitting the SED of each $1.6^{2}$ region. The histogram of the distribution of temperatures is shown in Figure 12. The mode of the temperature distribution was $\log T_{H}=6.42$ and the FWHM of the distribution was 6.30-6.56.

To within the uncertainties, the spatial distribution of the temperature of the hard component is uniform. The gradient from bottom to top in Figure 9 matches the scan pattern seen in the

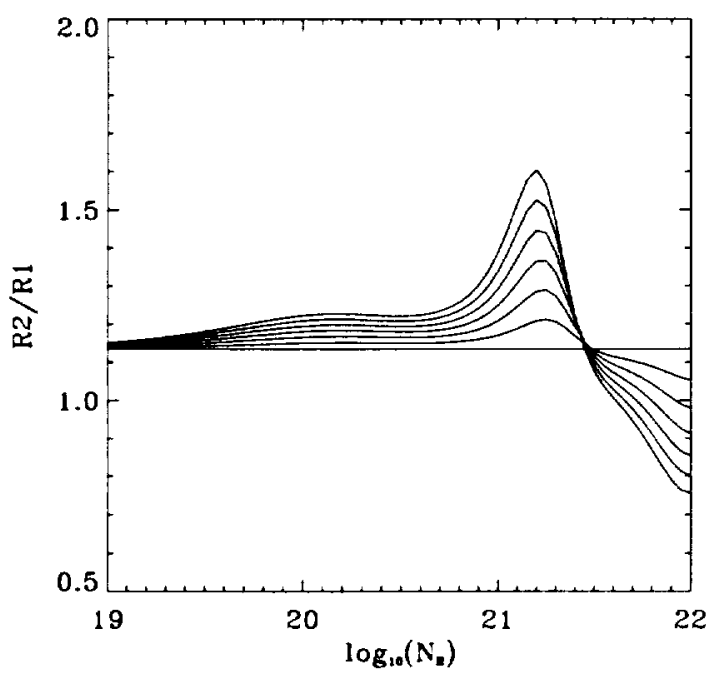

Fig. 11.- The derived R2/R1 ratio as a function of $N_{H}$ if $N_{H}$ were underestimated. We assumed the distant emission to have $\log T=6.15$ and measured the R2/R1 ratio of the deabsorbed emission when the absorbing column had been underestimated by (from bottom to top) $0 \%$ to $30 \%$ in steps of $5 \%$. 
RASS images; the pattern has an amplitude of $\Delta \log T \sim 0.1$.

\subsubsection{Comparisons}

Comparison of the components: If the TAE were produced by a single component, then one would expect the R12 and R45 bands for the TAE to be correlated. The lack of correlation is shown in Figure 13.

Comparison of one- and two-component models: Given the R1 and R2 measures of the TAE (from the local/distant separation), and the R4$\mathrm{R} T$ measures of the TAE (from direct RASS observation), we can construct the SED for each $1: 6^{2}$ region on the sky. We can then measure the relative goodness of fit for both the one- and twocomponent models.

To minimize the introduction of a bias through deabsorption, we created an absorbed SED from that part of the sky with $0.95<N_{H} / 10^{20}<1.15$ $\mathrm{cm}^{-2}$. For all bands we have taken the SED elements to be $I_{O}-\left(I_{L}+I_{E}\right)$ where $I_{E}$ is the contribution due to the absorbed EPL. We have then fit the two component model to each $1.6^{2}$ pixel using the previously derived temperatures, and determined the mean $\chi^{2}$ per fit, $\overline{\chi_{\nu}^{2}}=3.39(\nu=4)$.

The temperature of the single-component model was determined iteratively in a manner similar to that used for the two-component model. We fit a single-component model to the SED used for the two component fit. That temperature was then used to do the R1 and R2 band local/distant separation, and to redetermine the SED. The singlecomponent model was refit to the R1-R7 SED, and the procedure was repeated until sucessive fits produced the same value. We then found the temperature for which a single-component model had the best mean $\chi^{2}$ per fit, $\overline{\chi_{\nu}^{2}}=6.62$ $(\nu=5)$ at $\log T=6.20$. If the single-component model were correct, one would expect that the temperature produced by fitting the SED should be consistent with that found from the $R 2 / R 1$ ratio. Using $\log T=6.20$ as the TAE temperature in the local/distant separation, we find the unabsorbed R2/R1 band ratio to be 0.97 , equivalent to a Raymond \& Smith model temperature of $\log T=6.07$. Therefore, although our procedure attempts to make the temperatures found by SED fitting and by local/distant separation con-

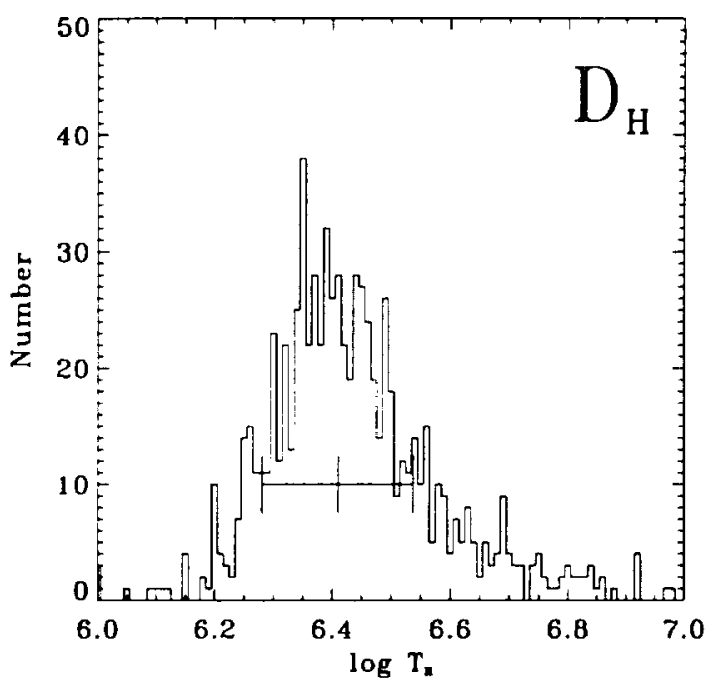

Fig. 12.- The distribution of the temperatures produced by fitting Raymond \& Smith models to the R4-R7 SED after the emission due to the LHB and the soft component of the TAE have been removed.

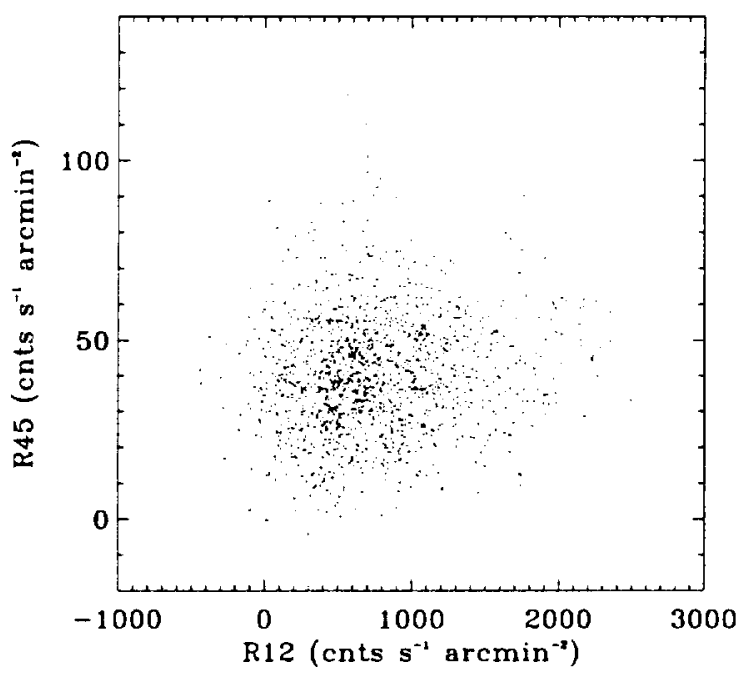

Fig. 13.- Correlation of the $\frac{1}{4} \mathrm{keV}$ (R12) band emission of the TAE and the $\frac{3}{4} \mathrm{keV}(\mathrm{R} 45)$ band emission of the TAE. The points are shown without error bars for clarity. 


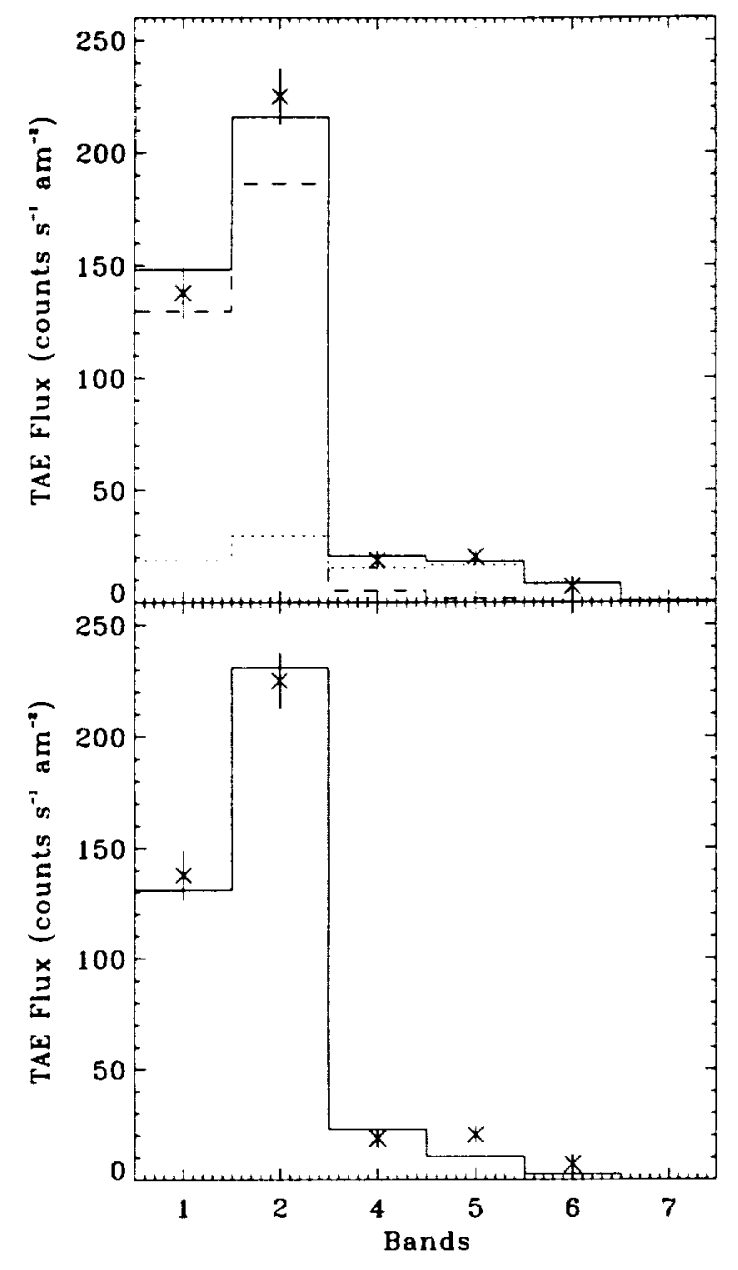

Fig. 14.- Fitting one- and two-component TAE models to the R1-R7 band SED. Both panels use a "mean" SED calculated from all regions where $0.95<N_{H} / 10^{20}<1.15$ and $\left(45^{\circ}<\ell<270^{\circ}, b>\right.$ $\left.55^{\circ}\right)$. The uncertainties are representative for individual $1: 6^{2}$ pixels. Top: The data are the $x$, the solid line is the best fit two-component TAE model, the dotted line is the hard component, the dashed line is the soft component. Bottom: The data are the $\times$, the solid line is the best fit onecomponent TAE model. Note that the SED used for the one-component model is slightly different from that used for the two-component model. See text for explanation. Note that the relative $\chi^{2}$ values given in the text were derived from all of the pixels within $\left(45^{\circ}<\ell<270^{\circ}, b>55^{\circ}\right)$ while the fits shown are to the mean of a subsample of the pixels used. sistent, no single-component model can be made consistent.

The two-component TAE model is clearly a significantly better fit. Results for a "mean" SED derived from a limited range of absorbing column density $\left(0.95<N_{H} / 10^{20}<1.15\right)$ can be seen for both models in Figure 14. The single-component temperature derived here is consistent with other single-component analyses. The relatively poor fit of the single-component model is due to forcing the SED fit to be consistent with the local/distant separation.

More Components?: The data are clearly insufficient to determine the existence of further components; any combination of thermal components that mimic the two derived here will work as well. The spatial distribution of the temperature of the hard component (Figure 9) suggests that that temperature may vary with position. Whether there is a single component with a spatially variable temperature, or several components with different temperatures must, at this point, remain a semantic distinction.

Conversely, one can ask if the hard thermal component is actually due to some component of the extragalactic background. If so, then the spectrum of the background must turn up at $\sim 1 \mathrm{keV}$, but turn back down before the $\frac{1}{4} \mathrm{keV}$ band; possible, but contrived.

It is likely that some portion of the hard thermal component is due to unresolved Galactic stars. Stellar X-ray emission is usually modeled by thermal plasmas with $\log T \sim 6.3-6.5$. A calculation of the X-ray flux due to stars, similar to that made by Guillout et al. (1996), shows that no more than $\sim 30 \%$ of the hard component flux can be due to unresolved stellar point sources. At high galactic latitudes, in directions of nominal diffuse background levels, only $\sim 25 \%$ of the $\frac{3}{4} \mathrm{keV}$ and 1.5 $\mathrm{keV}$ band flux which is not due to the LHB or the EPL can be due to unresolved stars.

Comparison of point source spectrum models: As noted in $\S 3.3 .1$, one must correct the RASS for point sources below the RASS removal limit but brighter than those discarded by Chen et al. (1997) when measuring the spectrum of the EPL. The spectrum of those sources is poorly understood in the $\frac{1}{4} \mathrm{keV}$ and $\frac{3}{4} \mathrm{keV}$ bands. The results presented above use $\Gamma=2.54$. for the point 
source correction. If a $\Gamma=1.46$ spectrum were used instead, the changes to the derived temperatures is minimal; $\log T_{L}=6.10, \log T_{S}=6.08$. and $\log T_{H}=6.39$ for $\Gamma=1.46$ vs. $\log T_{L}=6.10$, $\log T_{S}=6.06$, and $\log T_{H}=6.42$ for $\Gamma=2.54$.

Comparison of plasma models: From Table 1 it will be remembered that the model temperature derived depended, to some degree, upon the plasma model used. To some extent, this disagreement is immaterial; no plasma model has been able to reproduce the line spectrum of the diffuse X-ray background (Sanders et al. 1998; Vallerga \& Slavin 1998; Deiker et al. 1997). Therefore, the model temperatures derived here may be nothing more than convenient markers on an artificial but universally accessible scale. However, these markers certainly allow comparison of some measure of the relative temperatures of the gas.

Using XSPEC (Arnaud 1996) to fit MeweKaastra-Leidahl models to unabsorbed Raymond \& Smith models convolved with the ROSAT response, we find that

$$
\log T_{M K L} \sim 0.92+0.83 \log T_{R S} .
$$

Thus, the equivalent MKL temperatures will be systematically lower than the Raymond \& Smith values quoted here.

\subsubsection{The Diffuse Background Budget}

Table 2 presents the budget for the diffuse soft $\mathrm{X}$-ray background toward the north galactic pole (b $>85^{\circ}, 45^{\circ}<\ell<270^{\circ}$, with the region around the Coma cluster excluded). The line marked "Remainder" is the total observed flux from which has been subtracted the contributions from the LHB, the EPL, and the point source correction necessary for point sources not excluded from the RASS, but too bright to have been included in the Chen et al. (1997) formulation of the EPL. Hard and soft thermal components with $\log T_{H}=6.42$ and $\log T_{S}=$ 6.06 were fit to the remainder to determine their normalizations. The final line shows what portion of the remainder is due to unresolved stellar X-ray sources. It should also be noted from the work of Snowden et al. (2000) that of the remainder no more than $80 \times 10^{-6}$ counts $\mathrm{s}^{-1} \mathrm{arcmin}^{-2}$ in the $\frac{1}{4} \mathrm{keV}$ band can be truly extragalactic. (That is, after the removal of the EPL, the minimum flux observed originating from beyond the galactic $\mathrm{H} \mathrm{I}$ layer is $80 \times 10^{-6}$ counts s arcmin $^{-2}$.)

\subsubsection{Spectral Fitting Redux}

Using the knowledge gained above, one can return to the spectral fitting method. We have used three deep pointed PSPC exposures to test the consistency of our broad-band RASS results with more detailed spectral fitting. Whereas Pietz et al. (1998) selected pointings that were close together but had different absorbing columns, we used maps of the distant $\frac{1}{4} \mathrm{keV}$ emission to choose directions with different TAE intensities. We then searched the ROSAT archive at the HEASARC for pointed observations longer than $10 \mathrm{ks}$ in those directions, and which did not include clusters of galaxies or particularly bright point sources. Table 3 details these observations. The three observations, one exposure of the Lockman Hole deep survey, one of an AGN, and one of a star, were then prepared for spectral fitting. We first examined the light curves of the observations and eliminated times of bright scattered solar X-ray contamination, short-term enhancements (most likely a background due to auroral $\lambda$-rays), and longterm enhancements (the observations were relatively unaffected by the last). We examined images of the data and created an extraction region which included most of the area inside of the ring of the window-support structure but excluded the targets of the AGN and stellar observations. The same extraction region was used on all observation data sets. We binned the data into pulse-height channels with a minimum of 25 counts and created ancillary region files (ARFs).

XSPEC V.10 was used to fit the three observations simultaneously with several different models. We first fit the data with the two-component TAE model: an unabsorbed thermal spectrum for the LHB, two absorbed thermal spectra for the TAE, and the absorbed EPL. For the fit we fixed the power-law slope to the Chen et al. (1997) value of 1.46 but allowed the normalization to vary. (The fitted normalization was $12.1 \mathrm{keV} \mathrm{cm} \mathrm{cm}^{-2} \mathrm{~s}^{-1} \mathrm{sr}^{-1}$ $\mathrm{keV}^{-1}$.) The temperatures of the three thermal components were allowed to vary but they were constrained to be the same for all three data sets. The normalizations of the local component and the soft absorbed component were fit independently. for the three data sets but the normalizations of the hard thermal component and power law were constrained to be the same. The data were fit in the $0.12-1.90 \mathrm{keV}$ band. Table 4 lists the results, 
TABLE 2

DIFFUSE BACKGROUND BUdGET ${ }^{\mathrm{a}}$

\begin{tabular}{|c|c|c|c|c|c|c|}
\hline \multirow[t]{2}{*}{ Component } & \multicolumn{3}{|c|}{$\begin{array}{c}\text { Observed Fluxes } \\
10^{-6} \text { counts s}^{-1} \operatorname{arcmin}^{-2}\end{array}$} & \multicolumn{3}{|c|}{$\begin{array}{l}\text { Fluxes Before Absorption } \\
10^{-6} \text { counts s }^{-1} \text { arcmin }^{-2}\end{array}$} \\
\hline & $\mathrm{R} 12$ & $\mathrm{R} 45$ & $\mathrm{R} 67$ & $\mathrm{R} 12$ & $\mathrm{R} 45$ & $\mathrm{R} 67$ \\
\hline Total Observed & 1036.6 & 122.3 & 122.6 & & & \\
\hline LHB & 575.2 & 9.6 & 0.4 & & & \\
\hline EPL & 94.0 & 57.1 & 105.4 & 245.6 & 60.5 & 107.4 \\
\hline $\mathrm{PSC}^{\mathrm{C}}$ & -31.5 & 8.9 & 9.6 & -84.6 & 9.4 & 9.83 \\
\hline Remainder & 399.0 & 46.7 & 7.2 & & & \\
\hline Best Fit Soft ${ }^{d}$ & 349.8 & 8.9 & 0.3 & 1015.6 & 10.5 & 0.4 \\
\hline Best Fit Harde & 50.7 & 37.4 & 10.1 & 124.9 & 40.0 & 10.5 \\
\hline Stars ${ }^{f}$ & 9.0 & 9.7 & 4.3 & & & \\
\hline
\end{tabular}

${ }^{a} R A S S$ data taken from $b>85^{\circ}, 45^{\circ}<\ell<270^{\circ}$.

${ }^{b}$ Mean absorbing column was $1.32 \times 10^{20} \mathrm{~cm}^{-2}$.

'The point source correction described in the text, $\S 3.3 .1$

${ }^{\mathrm{d}}$ The best fit emmission measure was $7.3 \times 10^{-3} \mathrm{~cm}^{-6} \mathrm{pc}$.

${ }^{e}$ The best fit emmission measure was $2.3 \times 10^{-3} \mathrm{~cm}^{-6} \mathrm{pc}$.

${ }^{f}$ Unresolved stellar X-ray sources which contribute to the hard thermal component.

TABLE 3

Pointed Observations Used

\begin{tabular}{lccccc}
\hline \hline \multirow{2}{*}{ Observation } & \multirow{2}{*}{$N_{H}$} & $\ell$ & $b$ & \multicolumn{2}{c}{ Exposure(ks) } \\
& & & & Total & Useful \\
\hline rp900029a04 & $0.56 \times 10^{20}$ & 149.51 & 53.16 & 46.7 & 46.7 \\
rp700996n00 & $2.16 \times 10^{20}$ & 217.00 & 55.45 & 19.6 & 14.4 \\
rp200721n00 & $1.88 \times 10^{20}$ & 86.55 & 40.91 & 46.9 & 28.0 \\
\hline
\end{tabular}




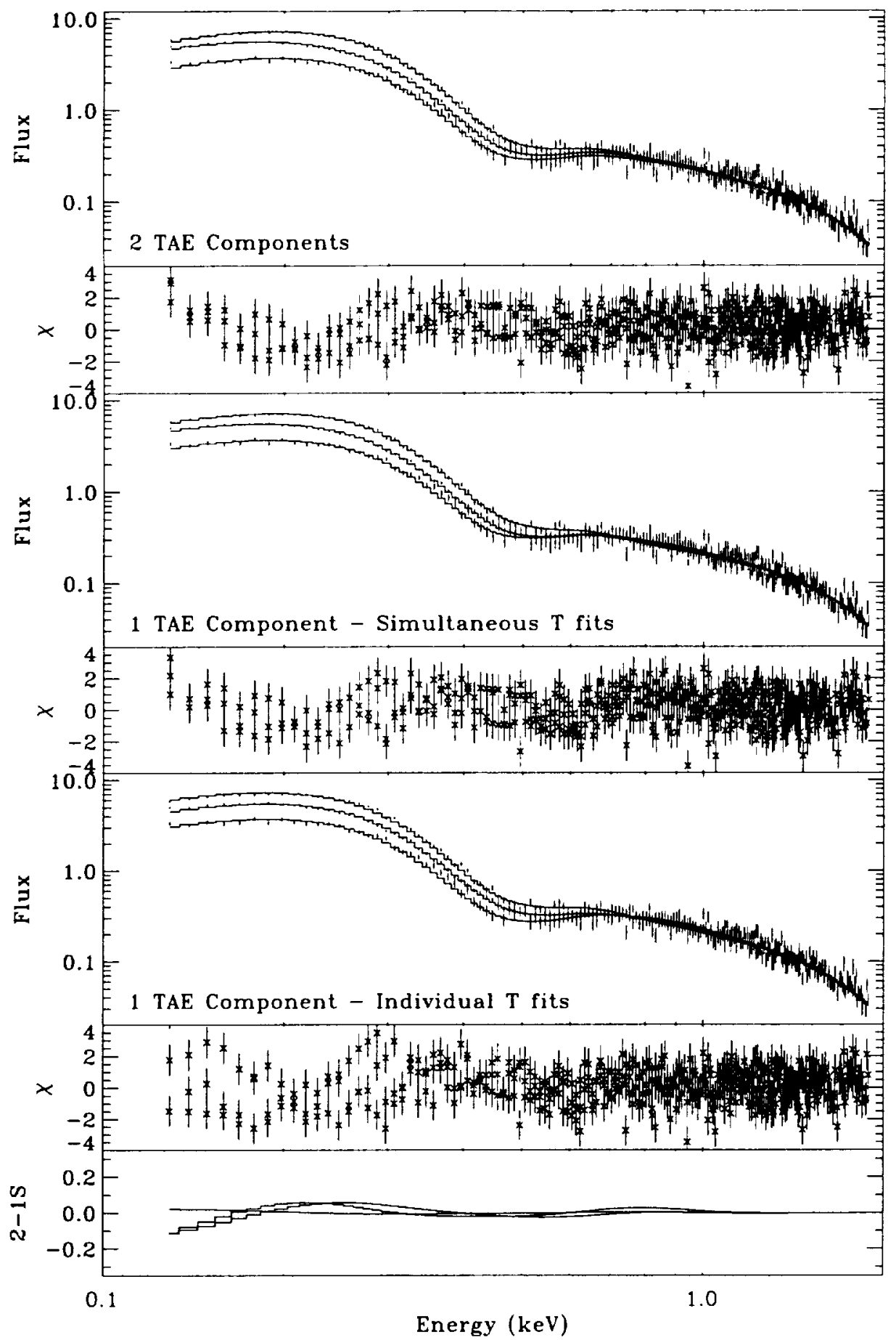

Fig. 15.- Simultaneous fits of three PSPC spectra from deep pointings. Top: Fit by a model containing the EPL, LHB and a two-component TAE. 2nd Panel: The residuals to the two-component fit. 3rd Panel: Fit by a model containing the EPL, LHB, and a one-component TAE; all the spectra are fitted with the same temperature TAE. 4th Panel: The residuals to the one component fit. 5th Panel: Fit by a model containing the EPL, LHB, and a one-component TAE; each spectrum is fitted with its own TAE temperature. 6th Panel: The residuals to the one-component fit. Bottom: The best fit two-component models minus the best fit one-component models, where the one-component models were required to have the same TAE temperature, i.e. the models in the third panel. 
TABLE 4

SPECTRAL FITS

\begin{tabular}{|c|c|c|c|c|c|c|c|c|}
\hline Model & $\log T_{L}$ & $N_{L}^{\mathrm{a}}$ & $\log T_{D 1}$ & $N_{D 1}^{a}$ & $\log T_{D 2}$ & $N_{D 2}{ }^{\mathrm{a}}$ & $\chi_{\nu}^{2}$ & $\nu$ \\
\hline \multirow[t]{3}{*}{2 Comp } & $6.10 \pm 0.02$ & $1.28 \pm 0.53$ & $6.00 \pm 0.02$ & $<0.77$ & $6.32 \pm 0.02$ & $0.64 \pm 0.24$ & 1.22 & 450 \\
\hline & & $0.73 \pm 0.14$ & & $1.72 \pm 0.60$ & & & & \\
\hline & & $2.61 \pm 0.04$ & & $4.14 \pm 0.89$ & & & & \\
\hline \multirow[t]{3}{*}{$1 \mathrm{Comp}^{\mathrm{b}}$} & $6.17 \pm 0.03$ & $1.76 \pm 0.04$ & $6.56 \pm 0.07$ & $<0.05$ & & & 1.39 & 452 \\
\hline & & $1.28 \pm 0.04$ & & $0.20 \pm 0.09$ & & & & \\
\hline & & $2.61 \pm 0.04$ & & $<0.05$ & & & & \\
\hline \multirow[t]{3}{*}{$1 \mathrm{Comp}^{\mathrm{c}}$} & $6.10 \pm 0.02$ & $1.28 \pm 0.24$ & $6.31 \pm 0.23$ & $0.64 \pm 0.3 j$ & & & 1.26 & 450 \\
\hline & & $0.09 \pm 0.14$ & $6.24 \pm 0.02$ & $1.25 \pm 0.42$ & & & & \\
\hline & & $1.58 \pm 0.26$ & $6.13 \pm 0.01$ & $3.01 \pm 0.71$ & & & & \\
\hline
\end{tabular}

${ }^{a}$ Units are $10^{-3}$ photons $\mathrm{cm}^{-2} \mathrm{~s}^{-1} \mathrm{keV}^{-1}$ in a solid angle of $8.54 \times 10^{-5} \mathrm{sr}$.

b The temperature of the absorbed thermal component was constrained to be the same for all data sets. The normalization of the extragalactic power law was fixed at the fitted value from the two-component model.

'The temperature of the absorbed thermal component was fitted independently for all the data sets.

which show a reasonable goodness of fit $\left(\chi_{\nu}^{2}=1.22\right.$ with $\nu=450$ ). The fitted values of the temperatures are consistent with our broad-band analysis.

We also fit the data with the one-component TAE model: an unabsorbed thermal spectrum for the LHB, one absorbed thermal component for the TAE, and the absorbed EPL. The temperatures of the thermal components and the normalization of the power law were constrained to be the same for all data sets but the normalizations for the thermal components were fit individually. With two fewer fitted parameters the reduced $\chi^{2}$ value increased from 1.22 to 1.38 . This rather significant reduction in the quality of the fit relative to the two-component model supports our claim that the one-component model cannot, in general, be fit simultaneously to multiple directions on the sky. Examination of the fitted parameters (Table 4) reveals a much higher temperature for the absorbed thermal component $(\log T \sim 6.56)$, and lower values for the normalizations. The fitting algorithm forced the local emission to accept a portion of the distant emission.

To examine the situation further, we allowed the temperature of the absorbed thermal compo- nent in the one-component model to be fit individually to each data set. This produced a considerable improvement in the $\chi^{2}$ value, to 1.26 , now with the same number of fitted parameters as the two-component model. However, the fitted values for the temperatures (Table 4) were completely inconsistent with a constant value (as expected with the large change in the quality of the fit). The brighter the $\frac{1}{4} \mathrm{keV}$ TAE the cooler the fitted temperature. This is exactly the expected result if the $\frac{1}{4} \mathrm{keV}$ intensity arises from a different emission component than the $\frac{3}{4} \mathrm{keV}$ intensity. Thus the two-component model is completely consistent with the detailed spectral fits of the three observations while the one-component model is not.

The results listed in Table 4 also demonstrate the ease with which the fitting algorithm can shift intensities and spectral parameters between one component and another. The choice of details used for the spectral model strongly affects the results which are obtained. The two separate fits to the one-component model provide an excellent example. In the first case, when the temperature of the TAE is constrained to be the same for all three data sets, the fitted temperature is very high and the fitted intensities are very low; such low intensi- 
ties are inconsistent with the variation of the halo emission which has been determined by shadowing experiments. This is clearly the case with the observation in the high-intensity direction (towards the Draco enhancement) where the fit provides a low value for the upper limit; flux from the TAE has been shunted to the LHB.

The difference between the temperature derived for the hot component by spectral fitting and that derived by SED fitting should not be considered too seriously; the two methods have very different systematics. Whereas, in the bicyclic iteration, the normalization and temperature of the LHB was very strongly constrained by the anticorrelation with the absorbing column, in the spectral fitting, the properties of the LHB are less strongly constrained, and so the fitting routine can distribute flux among the various components more freely, producing the best fit for those three particular spectra.

\subsection{The LHB}

Overview: The local/distant split that produces the R1 and R2 measures of the TAE also provides a measure of the emission due to the LHB. We find an LHB temperature of $\log T_{L}=6.10$ ( $F W H M=6.02-6.26)$ from the $\mathrm{R} 2 / \mathrm{R} 1$ band ratio 1.04 (FWHM $=0.890-1.220$ ). Although Snowden et al. (1998) and Snowden et al. (2000) contain detailed discussions of a similar analysis, as applied to the LHB, several points are worth noting. First, the temperature of the LHB, as derived by this analysis or by spectral fitting, depends sensitively upon the temperature found for the TAE. The total R1 and R2 band emission must be conserved, and the imposition of a given TAE temperature will determine the temperature to be found for the LHB. However, there are some checks upon the temperature of the LHB. Since, in the galactic plane, the column density in many directions is sufficient to block background $\frac{1}{4} \mathrm{keV}$ emission, one can determine the $R 2 / R 1$ ratio for the LHB without TAE contamination. After marshaling some essential data about the LHB, we will compare our measure of the LHB temperature to those in the literature.

Shape and Size: The shape of the LHB was determined by Snowden et al. (1998) from the all-sky deabsorption, but the size can not be set without knowing the emission measure within the LHB.
The standard scaling of the LHB is given by the observation of the molecular cloud MB.M 12, which produces a barely significant $\frac{1}{4} \mathrm{kel}^{\circ}$ shadow, implying that it is just barely within the LHB emission. The distance to MBM 12 has recently be redetermined to be $\sim 90 \mathrm{pc}$ by Sfeir et al. (1999). Thus, the high scaling of Snowden et al. (1998), $0.23 \mathrm{pc}\left(10^{-6} \text { counts s }^{-1} \text { arcmin }^{-2}\right)^{-1}$, should be used.

Internal Absorption: Sfeir et al. (1999) has compiled a homogeneous data set of 456 lines of sight with $\mathrm{Na} I \mathrm{D}$ absorption spectra with which they have mapped the Local Cavity. Their comparison of the Snowden et al. (1998) LHB contours to the Local Cavity shows that the bulk of the LHB fits inside the distance at which the cumulative column density is significantly less than $2 \times 10^{19} \mathrm{~cm}^{-2}\left(W_{\lambda}=10 \mathrm{~m} \AA\right)$; only some of the more peripheral regions of the LHB overlap directions/distances with such high column densities.

Absorption of the LHB emission is produced by the Local Interstellar Cloud (LIC) and several similar clouds within $\sim 5 \mathrm{pc}$. A mean absorbing column due to these features can be derived from Hutchinson et al. (1998) who showed that the mean absorbing column within $\sim 50 \mathrm{pc}$ was about $4 \times 10^{18} \mathrm{~cm}^{-2}$, about a factor of two greater than the highest LIC column densities (Lallement et al. 1995). We will use this value for the mean absorption of the LHB. It corresponds to approximately $3.6 \%$ absorption of the $\frac{1}{4} \mathrm{keV}$ emission. Other investigators have used greater columns; Sidher et al. (1996) used $6.6 \times 10^{18} \mathrm{~cm}^{-2}(\sim 5.9 \%$ absorption), Kerp (1994) and Pietz et al. (1998) used $1 \times 10^{19} \mathrm{~cm}^{-2}$ ( $\sim 8.7 \%$ absorption), and Parmar et al. (1999) used $7 \times 10^{19} \mathrm{~cm}^{-2}(\sim 43 \%$ absorption). The last of these values is completely incompatible with optical absorption line studies.

Temperature: The model temperature of the LHB can be determined from the R2/R1 (or $\mathrm{R} 2 / \mathrm{R} 1 \mathrm{~L}$ ) ratio in a direction in which absorption by the galactic ISM blocks $\frac{1}{4} \mathrm{keV}^{\prime}$ emission from more distant sources. Figure 16 demonstrates the difficulty of this problem. Numerous strong features visible in the $\frac{3}{4} \mathrm{keV}$ band, mostly supernovae and super-bubbles, penetrate the galactic absorption to contaminate the local emission, and increase the $\mathrm{R} 2 / \mathrm{R} 1$ ratio. One can calculate the hardness ratio in a large window towards the anticenter in between known sources 


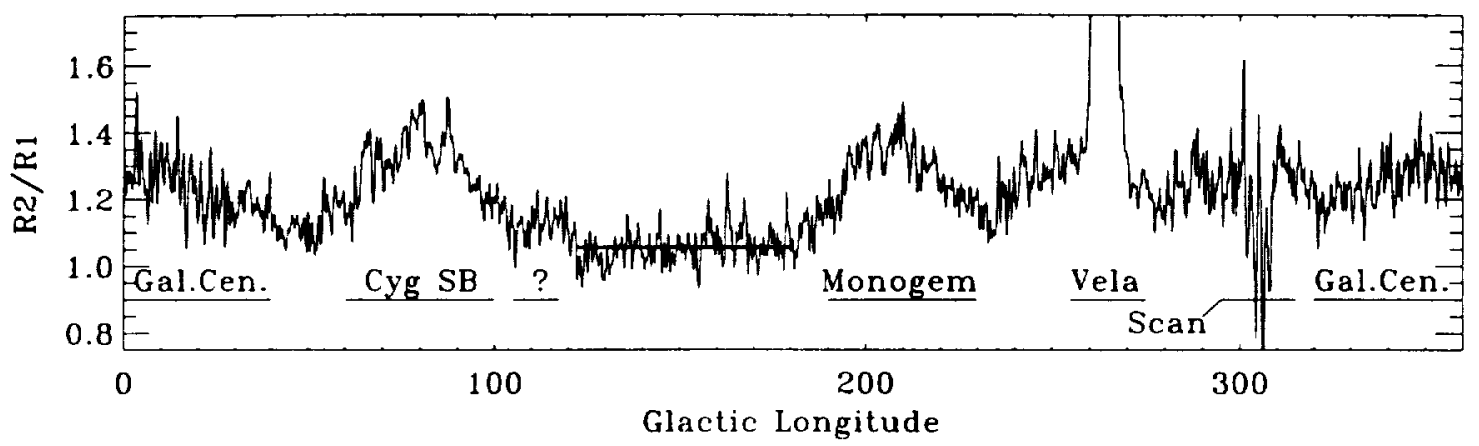

Fig. 16.-- The $\frac{1}{4} \mathrm{kel}$ hardness ratio as a function of galactic longitude for the region within $5^{\circ}$ of the galactic plane. Brighter, hotter, absorbed features that "leak" into the R12 band are labeled. "Scan" refers to region with poor exposure due to a missing scan. The horizontal bar overlaying the data shows the mean hardness ratio over the given ranges of galactic longitude.

of contamination, and one finds that the temperature is $\sim \log T=6.10(\mathrm{R} 2 / \mathrm{R} 1=1.057)$. The temperature of the LHB towards the galactic center does seem to be higher (Snowden et al. 1990b, 2000 ), but the contamination by the galactic bulge "leaking" through the absorption does not allow a determination with this data set.

One might think to avoid most sources of contamination by sampling just off of the galactic plane. The only region that is near the galactic plane and has a substantial absorbing column $\left(10^{21}<N_{H}<2.0 \times 10^{21} \mathrm{~cm}^{-2}\right)$ is the region of the Taurus-Auriga molecular clouds. Absorption line data place these clouds at $\sim 120 \mathrm{pc}$, so there is little room for extra emission components between the LHB (radius $\sim 100 \mathrm{pc}$ in this direction) and the absorbing clouds. A sample of the $R A S S$ of $\sim 360$ square degrees yields a $\mathrm{R} 2 / \mathrm{R} 1=$ $1.02 \pm 0.01$, or an equivalent Raymond \& Smith model temperature of $\log T=6.09 \pm 0.01$

Thus, the current analysis produces an LHB temperature through broad-band ratios that is consistent with independent measures also made with broad band ratios. The significantly lower temperatures found by the spectral fitting of past analyses are due to the need for a much higher temperature TAE, when one assumes that the TAE is due to a single thermal component.

\section{Discussion}

Historical Background: The discovery of the soft X-ray emission that we have come to associate with the LHB occurred as the result of attempts to measure the soft extrapolation of the extragalactic power law (Bowyer et al. 1968; Bunner et al. 1969: Davidsen et al. 1972). The ubiquity of the $\frac{1}{4} \mathrm{keV}$ emission was a mystery that was not to be solved by the discovery of instrumental effects or incorrect modeling of the particle background, though these problems remain. (See, for example, the discussion of the Long Term Enhancements and the earth as a "flaming comet" in Cox 1998.) The $\frac{1}{4} \mathrm{keV}$ emission anti-correlation with the total neutral hydrogen column led to two main schools of thought. The first, the absorption model (Bowyer et al. 1968; Bunner et al. 1969; Davidsen et al. 1972), was that the ubiquitous emission was extragalactic and absorbed, though it was found that the absorption cross-sections could not produce the observed dynamic ranges (McCammon et al. 1976; Long et al. 1976; Burrows et al. 1984) or band ratios (Bloch et al. 1986; Burrows 1989; Juda et al. 1991). The second, the displacement model (Sanders et al. 1977; Cox \& Snowden 1986; Cox \& Reynolds 1987; Snowden et al. 1990a), was that the emission was proportional to the unabsorbed path length, which was roughly anti-correlated to the total column. There was a third model, the embedded cloud model, Jakobsen \& Kahn (1986), based somewhat on the McKee \& Ostricker (1977) model of the ISM, that suffered from many of the same problems as did the absorption model (Burrows 1989). The advent of X-ray shadows (Snow- 
den et al. 1991; Burrows \& Mendenhall 1991), observed early in the ROSAT era, showed that both models were, to some extent, correct.

It was clear that the soft X-ray background was composed of at least three components, the LHB, the EPL, and some component beyond the galactic absorption, which we have called the TAE. Although the Draco shadows $\left(\ell=89^{\circ}-91^{\circ}, b=\right.$ $38^{\circ}-40^{\circ}$ ) were the first glimpse of a hot galactic halo, it was not the long-theorized galactic corona (Spitzer 1956); the $\frac{1}{4} \mathrm{keV}$ TAE was too patchy (Burrows \& Mendenhall 1991; Snowden et al. 1991, 1994a). It was suggested that the $\frac{1}{4} \mathrm{keV}$ and $\frac{3}{4} \mathrm{keV} \mathrm{TAE}$ were due to different components (Burrows \& Mendenhall 1991) as the $\frac{3}{4} \mathrm{keV}$ emission was much smoother than the $\frac{1}{4} \mathrm{keV}$ emission. Like the infamous Leiden-Groningen H I $21 \mathrm{~cm}$ data split that produced the artificial separation between high and intermediate velocity clouds, the $R A S S$ was split between the $\frac{1}{4} \mathrm{keV}$ and $\frac{3}{4} \mathrm{keV}$ bands and analysed by different groups.

Summary of the Argument: The current work attempts to effect the obvious synthesis of the two bands. Our results have shown that the TAE is composed of at least two thermal components, perhaps more. The result is based on five arguments: 1) That the TAE of the R12 and R45 bands are not correlated, 2) That no single component can produce both the observed anti-correlation of emission and absorbing column and a consistent SED, 3) That the two-component models fit the SED better than any single-component model, 4) That single component models are not acceptable because they do not allow the LHB to have a temperature that is consistent with that measured by the $\mathrm{R} 2 / \mathrm{R} 1$ ratio in the galactic plane where there is no "leaking" background emission, and 5) That the two component model is more consistent with detailed spectral fitting than are the single component models. The argument that the $\frac{3}{4} \mathrm{keV}$ emission is much smoother than the $\frac{1}{4} \mathrm{keV}$ emission is no more than suggestive. With the exception of such strong features as the Draco enhancement, a single-temperature model, in order to get the proper R45/R12, will necessarily scale down the structures seen in the R12 band. Since much of the emission in the R45 band is due to the EPL, the small variations due to the TAE would become difficult to detect in the R45 band.

Although we posit two (or more) components to the TAE, the components are not neatly segregated into the $\frac{1}{4} \mathrm{keV}$ and $\frac{3}{4} \mathrm{keV}$ bands; $\sim 22 \%$ of the $\frac{3}{4} \mathrm{keV}$ band flux (before absorption) is produced by the soft component, while $\sim 12 \%$ of the $\frac{1}{4} \mathrm{keV}$ band flux (also before absorption) is produced by the hard component. Because the soft component does not have a significant trend with galactic longitude, global analyses that utilize only the R4-R7 bands, such as Wang (1997), will not be significantly affected by our analysis.

Global Properties of the TAE: The mean surface brightness of the soft TAE component is $\sim 816$ counts $\mathrm{s}^{-1}$ arcmin $^{-2}$ or $\sim 3.3 \times 10^{-8} \mathrm{ergs} \mathrm{cm}^{-2} \mathrm{~s}^{-1}$ $\mathrm{sr}^{-1}$ over the 0.1-2.0 keV range; for the hard component, $\sim 155$ counts s$^{-1}$ arcmin $^{-2}$ or $\sim 1.2 \times 10^{-8}$ ergs $\mathrm{cm}^{-2} \mathrm{~s}^{-1} \mathrm{sr}^{-1}$ over the $0.1-2.0 \mathrm{keV}$ range. Even away from Loop I $\left(\ell=330^{\circ} \pm 60^{\circ}\right)$ and the Draco enhancement $\left(\ell \sim 90^{\circ}\right)$, the soft component is quite mottled, though the azimuthally averaged distribution is quite constant. The hard component, away from Loop I, is smooth (see Figure 9).

These characteristics suggest that the hard component might be identified with the galactic corona or a galactic halo in hydrostatic equilibrium (Wang 1997; Pietz et al. 1998), though that identification is by no means certain. If the hard component is the corona, with what is the soft component to be identified?

The missing piece in halo studies is often the distance to the emission, and this study is no exception. Several studies have set the $\frac{1}{4} \mathrm{keV}$ terminus a quo. The $\frac{1}{4} \mathrm{keV}$ shadowing clouds in Draco have distances $z>180 \mathrm{pc}$ (Lilienthal et al. 1991). Benjamin et al. (1996) found distances of $z \sim 285 \pm 75 \mathrm{pc}$ to an infrared cirrus cloud in the Lockman hole that shadows the distant X-ray emission. The determination of the terminus ad quem is more difficult. The attempts to use high and intermediate velocity clouds are difficult as the disk absorption is much stronger, and there have been suggestions that the high velocity clouds may have X-ray emitting bow-shocks. A search for $\frac{1}{4} \mathrm{keV}$ shadows due to high velocity complex $M$ $(1.5<z<4.4 \mathrm{kpc}$ ) was equivocal (Herbstmeier et al. 1995). A trivial upper limit is set by the shadowing of the extragalactic background by several galaxies (Barber \& Warwick 1994; Snowden \& Pietsch 1995; Barber et al. 1996; Cui et al. 1996). Distance determinations for the $\frac{3}{4} \mathrm{keV}$ emission by shadowing are even more difficult as high and 
intermediate velocity clouds do not have sufficient column density to produce a measurable shadow:

External Galaxies: Observations of external galaxies provide further clews to the distribution of the TAE. The $\frac{1}{4} \mathrm{kel}$ flux due to the TAE (both components) is $\sim 750$ counts $\mathrm{s}^{-1} \operatorname{arcmin}^{-2}$, while the $\frac{3}{4} \mathrm{keV}$ flux is $\sim 40$ counts $\mathrm{s}^{-1} \operatorname{arcmin}^{-2}$. Thus, despite the absorption by our own galaxy, the closer external galaxies should have detectable halos.

The X-ray halos of face-on galaxies are contaminated by the point sources in the disk, but this contamination has diminished as resolution has improved. The best study to date is that of the closest face-on Milky Way analogue, M101, which was observed by the ROSAT PSPC (Snowden \& Pietsch 1995). If we use the relative $D_{25}$ values for the two galaxies to scale to the equivalent of the galactocentric radius of the sun, a radius of $\sim 8.5$, and if we assume that the entire M101 flux, after point source removal, is due to the halo, we find 1570 counts s$^{-1} \operatorname{arcmin}^{-2}$ at $\frac{1}{4} \mathrm{keV}$, while the flux at $\frac{3}{4} \mathrm{keV}$ at that radius is indistinguishable from the background. Significant $\frac{1}{4} \mathrm{keV}$ flux $(\sim 350$ counts $\mathrm{s}^{-1} \operatorname{arcmin}^{-2}$ ) extends to $\sim 20^{\prime}$. (The scale length of the $\frac{1}{4} \mathrm{keV}$ emission is $\sim 0.4 D_{25} / 2$, and the scale length of the $\frac{3}{4} \mathrm{keV}$ emission would appear to be similar.) Thus, both the strength and extent of the soft component of the TAE is consistent with that observed in M101. The temperature determined from the $\mathrm{R} 2 / \mathrm{R} 1$ ratio for the M101 halo is, however, cooler, $\log T=5.86$.

Edge-on spiral galaxies for which extra-planar $\mathrm{X}$-ray emission has been detected are often strong star-burst galaxies. Three non-starburst edge-on spiral galaxies have ROSAT-detected $\mathrm{X}$-ray halos: NGC 891, NGC 4631, and NGC 4565. Of these, NGC 4565 has the lowest stellar formation rate (SFR, as measured by $L_{F I R} / D_{25}^{2}$ ) and has no detected extended diffuse ionized gas (DIG). Vogler et al. (1996) detected some extraplanar diffuse X-ray emission, but were unable to determine a scale-height, and could only say that the temperature was consistent with a few times $10^{6} \mathrm{~K}$ gas. NGC 4631, which has a higher SFR, is a disturbed galaxy that may be interacting (Weliachew et al. 1978; Rand et al. 1992; Rand \& Stone 1996; Donahue et al. 1995). It has a strong nuclear star formation, and nuclear Ha filaments, which may be related to a $\frac{1}{4} \mathrm{keV}$ emission region. The $\frac{3}{4} \mathrm{keV}$ emission has a HWHM $z$-extent of almost $3 \mathrm{kpc}$, a radial extent of about $3 \mathrm{kpc}\left(0.25 D_{25} / 2\right)$, and a temperature $\log T_{R S}=6.48$ (Wang et al. 1995 ) or $\log T_{M K L}=6.31$ (Dahlem et al. 1998). NGC 891, which has an even higher SFR, has a more uniformly extended $\mathrm{X}$-ray halo; the $\frac{3}{4} \mathrm{keV}$ emission has a HWHM $z$-extent of $2.1 \mathrm{kpc}$, a radial scale length of $4.1 \mathrm{kpc}\left(0.3 D_{25} / 2\right)$, and a temperature of $\log T=6.54$ (Bregman \& Houk 1997).

The only extragalactic detection (in an edge-on spiral) of a component that might be similar to the soft component of the TAE is that by Wang et al. (1995) in NGC 4631, where they detect a component with $\log T<5.78$. Dahlem et al. (1998) also detect a component with $\log T=5.76$, but Read et al. (1997) do not. Such a soft component could not be detected in NGC 891 due to the higher Galactic absorption. Lack of detection of the soft TAE component in external galaxies is not surprising; it is subject to strong absorption by both the Milky Way and the host galaxy. If the soft component has a lower scale-height than the hard component, then not only will the absorption by the host galaxy be important, but the ROSAT resolution would not allow separation of the soft TAE from disk emission.

Pure Speculation: Both NGC 4631 and NGC 891 have SFRs, based upon $L_{F I R} / D_{25}^{2}$, that are similar to that of the Milky Way (Rand 1998). Both NGC 4631 and NGC 891 have halo temperatures that are consistent with the $\log T=6.42$ that we have found for the hot component of the TAE. Given the similarities of halo temperature, and that the extent of the extra-planar diffuse ionized gas is roughly dependent upon the SFR, it seems likely that the smooth hard component of the TAE has $z$-scale heights $\sim 2 \mathrm{kpc}$, somewhat smaller than the $4 \mathrm{kpc}$ exponential scale height fitted by Pietz et al. (1998). Both M101 and NGC 891 imply that the hard component should have a radial scale length $\sim 0.35 D_{25} / 2$. or $\sim 4$ $\mathrm{kpc}$, which is much smaller than the $15 \mathrm{kpc}$ scale length fitted by Pietz et al. (1998), but implies a much more disk-like distribution than the model of Wang (1997).

The $\frac{3}{4} \mathrm{keV}$ emission in the Milky Way is quite symmetric, the mean flux in the south being very similar to that of the north, the emission is quite symmetric in NGC 891, and the asymmetry of NGC 4631 is thought to be an inclination effect 
(Wang et al. 1995). With a disk-like distribution, this symmetry would argue that the hard component is hot gas in hydrostatic equilibrium, where the symmetry across the disk is enforced by the gravitational potential.

The soft component, however, is very different. The soft component is patchy, and there is a distinct north-south asymmetry in the $\frac{1}{4} \mathrm{keV}$ TAE; the northern hemisphere is about 1.9 times stronger than the southern hemisphere (Snowden et al. 1998). and the temperature of the soft component in the southern hemisphere may be lower than that of the north (Snowden et al. 1998) as is implied by Figure 11 of Snowden et al. (2000). These properties suggest that the soft component is the result of local phenomena. Perhaps the soft component is composed of the plumes of hot gas ejected from galactic chimneys. Snowden et al. (1998) suggested that the Draco enhancement was the extraplanar breakout of the Cygnus superbubble, though the intervening mid-latitude absorption makes testing this model difficult. (It is interesting to note that such a model suggests that the Draco emission occurs to $z \sim 2 \mathrm{kpc}$.) However, as the $\frac{1}{4} \mathrm{keV} \mathrm{TAE}$ is not restricted to lower latitudes any local phenomenon must be fairly uniformly distributed or must send gas to fairly high $z$. Shelton (1998) has suggested that supernovae remnants in the halo might dominate the soft $\mathrm{X}$ ray emission and produce the patchy structure.

As has been pointed out by Gehrels \& Williams (1993) in the context of stellar coronae, the cooling curve for a hot thin plasma has two local minima at temperatures that would emit in the soft Xray range. Of course, the exact temperatures of those minima ( $\log T=5.9$ and 6.6) do not agree particularly well with the observed temperatures ( $\log T=6.06$ and 6.42). Reference to Schmutzler \& Tscharnuter (1993) shows that the location of the minima of the cooling curve are sensitive to the metallicity and previous ionization history of the gas. It is only suggestive that the minima of the cooling curve may correspond to the temperatures of the dominant components. In this case, the ubiquity of the soft component suggests that the soft component may represent some part of the hard component at a more advanced age.

Future Work: Determining the true thermal structure of the galactic halo will be difficult. Next generation $\mathrm{X}$-ray instruments will have lower background contamination, but typically will have small fields-of-view, so using angular variations caused by absorption to separate local and distant components will be more difficult. Although spectral resolution will be good at $\frac{3}{4} \mathrm{keV}$, the spectral resolution below the carbon edge, where the LHB and the soft component of the TAE has most of their emission, remains poor. Experience with the DXS has shown that given high spectral resolution, understanding the spectrum of the LHB is still difficult, and the parameter space allowed by non-equilibrium plasma codes is large.

Attention will also need to be given to incorporating the X-ray TAE into our understanding of the halo optical/UV emission/absorption line ratios. Many line ratios are adequately explained by photoionization due to massive disk stars, whose ionizing radiation is absorbed and hardened by passage through the gaseous disk (see, for example, the review by Rand 1998). However, there are still outstanding difficulties, such as the run of line ratios with $z$-height, in which the $X$-ray halo may play a part.

We would like to thank Robin Shelton, Ian George, Richard Mushotzky, Peter Teuben, Richard J. Rand, and Sylvain Veilleux for several interesting discussions. This study was funded in part by the NASA ADP program. 


\section{REFERENCES}

Arabadjis, J. S. \& Bregman, J. X. 1999, ApJ, 510, 806 , submitted

Arendt, R. G., Odegard, N., Weiland, J. L., Sodroski, T. J., Hauser, M. G., Dwek, E., Kelsall, T., Modeley, S. H., Silverberg, R. F., Leisawitz, D., Mitchel, K., Reach, W. T., \& Wright, E. L. 1998, ApJ, 508, 74

Arnaud, K. A. 1996, in Astronomical Data Anal?sis Software and Systems V, ed. G. Jacoby \& J. Barnes, 17

Barber, C. R., Roberts, T. P., \& Warwick, R. S. 1996, MNRAS, 282, 157

Barber, C. R. \& Warwick, R. S. 1994, MNRAS, 267,270

Benjamin, R. A., Venn, K. A., Hiltgen, D. D., \& Sneden, C. 1996, ApJ, 464, 836

Bloch, J. J., Jahoda, K., Juda, M., McCammon, D., Sanders, W. T., \& Snowden, S. L. 1986, ApJ, 308, L59

Bowyer, C. S., Field, G. B., \& Mack, J. E. 1968, Nature, 217, 32

Bregman, J. N. \& Houk, J. C. 1997, ApJ, 485, 159

Bunner, A. N., Coleman, P. L., Kraushaar, W. L., McCammon, D., Palmieri, T. M., Shilepsky, A., \& Ulmer, M. 1969, Nature, 233, 1222

Burrows, D. N. 1989, ApJ, 340, 775

Burrows, D. N., McCammon, D., Sanders, W. T., \& Kraushaar, W. L. 1984, ApJ, 287, 208

Burrows, D. N. \& Mendenhall, J. A. 1991, Nature, 351,629

Chen, L.-W., Fabian, A. C., \& Gendreau, K. C. 1997. MNRAS, 285, 449

Cox, D. P. 1998, in The Local Bubble, ed. D. Breitschwerdt \& M. Freyberg, 121-128

Cox, D. P. \& Reynolds, R. J. 1987, ARA\&A, 28, 303

Cox, D. P. \& Snowden, S. L. 1986, Advances in Space Research, 6, 97
Cui, W., Sanders, W. T., McCammon, D., Snowden, S. L., \& Womble, D. S. 1996, ApJ, 468, 117

Dahlem, M., Weaver, K. A., \& Heckman, T. M. 1998, ApJS, 118, 401

Davidsen, A., Shulman, S., Fritz, G., Meekins, J. F., Henry, R. C. \& Friedman, H. 1972, ApJ, 177,629

Deiker, S., Kelley, R. L., Lesser, A., McCammon, D., Porter, F. S., Stahle, W. T. S. C. K., \& Szymkowiak, A. E. 1997, in Proceedings of the Seventh International Workshop on Low Temperature Detectors, 113

Domgörgen, H. \& Mathis, J. S. 1994, ApJ, 428, 647

Donahue, M., Aldering, G., \& Stocke, J. T. 1995, ApJ, 450, L45

Gehrels, N. \& Williams, E. D. 1993, ApJ, 418, L25

Guillout, P., Haywood, M., Motch, C., \& Robin, A. C. $1996, A \& A, 316,89$

Hartmann, D. \& Burton, W. B. 1997, Atlas of Galactic Neutral Hydrogen (Cambridge University)

Hasinger, G., Burg, R., Giacconi, R., Hartner, G., Schmidt, M., Trümper, J., \& Zamorani, G. 1993, A\&A, 275, 1

Hasinger, G., Burg, R., Giacconi, R., Schmidt, M., Trümper, J., \& Zamorani, G. 1998, A\&A, 329, 482

Herbstmeier, U., Mebold, U., Snowden, S. L., Hartmann, D., Burton, W. B., Moritz, P., Kalberla, P. M. W., \& Egger, R. 1995, A\&A, 298,606

Hutchinson, I. B., Warwick, R. S., \& Willingale, R. 1998, in The Local Bubble, ed. D. Breitschwerdt \& . M. Freyberg, 283-286

Jakobsen, P. \& Kahn, S. M. 1986, ApJ, 309, 682

Juda, M., Bloch, J. J., Edwards, B. C., McCammon, D., Sanders, W. T., Snowden, S. L., \& Zhang, J. 1991, ApJ, 367, 182

Kerp, J. 1994, A\&A, 289, 597 
Kerp, J., Burton, W. B., Egger, R., Freyberg, M. J., Hartmann, D., Kalberla, P. M. W., Mebold, U., \& Pietz, J. 1999, A\&A, 342, 213

Kuntz, K. D. 2000, PhD thesis, University of Maryland

Kuntz, K. D. \& Snowden, S. L. 1998, Cookbook for Analysis Procedures for ROSAT XRT Observations of Extended Objects and the Diffuse Background, Part II: Mosaics, Tech. rep., NASA/GSFC

Lallement, R., Ferlet, R., Lagrange, A. M., Lemoine, M., \& Vidal-Madjar, A. 1995, A\&A, 304,461

Lampton, M., Margon, B., \& Bowyer, S. 1976, ApJ, 208, 177

Landini, M. \& Monsignori-Fossi, B. C. 1990, A\&AS, 82, 229

Laor, A., Fiore, F., Elvis, M., Wilkes, B. J., \& McDowell, J. C. 1997, ApJ, 477, 93

Lilienthal, D., Wennmacher, A., Herbstmeier, U., \& Mebold, U. 1991, A\&A, 250, 150

Long, K. S., Agrawal, P. C., \& Garmire, G. P. 1976, ApJ, 206, 411

McCammon, D., Meyer, S. S., Sanders, W. T., \& Williamson, F. O. 1976, ApJ, 209, 46

McKee, C. F. \& Ostricker, J. P. 1977, ApJ, 218, 148

Mewe, R., Gronenschild, E., \& van den Oord, G. H. J. 1985, A\&A, 62, 197

Miyaji, T., Ishisaki, Y., Ogasaka, Y., Ueda, Y., Freyberg, M. J., Hasinger, G., \& Tanaka, Y. 1998, A\&A, 334, L13

Parmar, A. N., Guainazzi, M., Oosterbroek, T., Orr, A., Favata, F., Lumb, D., \& Malizia, A. 1999, A\&A, submitted

Pietz, J., Kerp, J., Kalberla, P., Burton, W. B., Hartmann, D., \& Mebold, L. 1998, A\&A, 332, 55

Rand, R. J. 1998, Proc. Astron. Soc. Aust., 15, 106
Rand, R. J., Kulkarni, S. R., \& Hester, J. J. 1992, ApJ, 396, 97

Rand, R. J. \& Stone, J. M. 1996, AJ, 111, 190

Raymond, J. C. \& Smith, B. W. 1977, ApJS, 35

Reach, W. T., Wall, W. F., \& Odegard, N. 1998, ApJ, 507, 507

Read, A. M., Ponman, T. J., \& Strickland, D. K. 1997, MNRAS, 286, 626,668

Reynolds, R. J. 1991, in The Interstellar Disk-Halo Connection in Galaxies, ed. H. Bloemen, 67-76

Sanders, W. T., Burrows, D. N., McCammon, D., \& Kraushaar, W. L. 1983, in Supernova Remnants and their X-ray Emission, ed. J. Danziger \& P. Gorenstien, 361-365

Sanders, W. T., Edgar, R. J., Liedahl, D. A., \& Morgenthaler, J. P. 1998, in The Local Bubble, ed. D. Breitschwerdt \& M. Freyberg, 83-90

Sanders, W. T., Kraushaar, W. L., Nousek, J. A., \& Fried, P. M. 1977, ApJ, 217, L87

Schlegel, D. J., Finkbeiner, D. P., \& Davis, M. 1997, ApJ, 500, 525

Schmutzler, T. \& Tscharnuter, W. M. 1993, ApJ, 273,318

Sfeir, D., Lallement, R., Crifo, F., \& Welsh, B. Y. 1999, A\&A, submitted

Shelton, R. L. 1998, ApJ, 504, 785

Sidher, S. D., Summer, T. J., Quenby, J. J., \& Gambhir, M. 1996, A\&A, 305, 308

Snowden, S. L., Cox, D. P., McCammon, D., \& Sanders, W. T. 1990a, ApJ, 354, 211

Snowden, S. L., Egger, R., Finkbeiner, D., Freyberg, M. J., \& Plucinsky, P. P. 1998, ApJ, 493, 715

Snowden, S. L., Egger, R., Freyberg, M. J., McCammon, D., Plucinsky, P. P., Sanders, W. T., Schmitt, J. H. M. M., Trümper, J., \& Voges, W. 1997, ApJ, 485, 125

Snowden, S. L., Freyberg, M. J., Kuntz, K. D., \& Sanders, W. T. 2000, ApJS, in press 
Snowden, S. L., Freyberg, M. J., Plucinsky, P. P., Schmitt, J. H. M. M., J. Trümper, J., Voges, W., Edgar, R. J., McCammon, D., \& Sanders, W. T. 1995, ApJ, 454, 643

Snowden, S. L., Hasinger, G., Jahoda, K., Lockman, F. J., McCammon, D., \& Sanders, W. T. 1994a, ApJ, 430, 601

Snow.den, S. L. \& Kuntz. K. D. 1998, Cookbook for Analysis Procdures for ROSAT XRT Observations of Extended Objects and the Diffuse Background, Part I: Individual Observations, Tech. rep., NASA/GSFC

Snowden, S. L., McCammon, D., Burrows, D. N., \& Mendenhall, J. A. 1994b, ApJ, 424, 714

Snowden, S. L., McCammon, D., \& Verter, F. 1993, ApJ, 409, L21

Snowden, S. L., Mebold, U., Hirth, W., Harbstmeier, U., \& Schmitt, J. H. M. M. 1991, Science, 252,1529

Snowden, S. L. \& Pietsch, W. 1995, ApJ, 452, 627

Snowden, S. L., Schmitt, J. H. M. M., \& Edwards, B. C. 1990 b, ApJ, 364, 118

Spitzer, L. 1956, ApJ, 124, 20

Vallerga, J. \& Slavin, J. 1998, in The Local Bubble, ed. D. Breitschwerdt \& M. Freyberg, 79-82

Vogler, A., Pietsch. W., \& Kahabka, P. 1996, A\&A, 305, 74

Wakker, B. P. \& Boulanger, F. 1986, A\&A, 170, 84

Wang, Q. D. 1997, in The Local Bubble, ed. D. Breitschwerdt \& M. Freyberg, 503-512

Wang, Q. D., Walterbos, R. A. M., Steakley, M. F., Norman, C. A., \& Braun, R. 1995, ApJ, 439,176

Weliachew, L., Sancisi, R., \& Guélin, M. 1978, A\&A, 65,37

This 2-column preprint was prepared with the AAS IATEX macros 5.0 . 